\title{
Brain Tumor Regulates Neuromuscular Synapse Growth and Endocytosis in Drosophila by Suppressing Mad Expression
}

\author{
Wenwen Shi, ${ }^{1 \star}$ Yan Chen, ${ }^{1 \star}$ Guangming Gan, ${ }^{2}$ Dan Wang, ${ }^{1}$ Jinqi Ren, ${ }^{1}$ Qifu Wang, ${ }^{1}$ Zhiheng Xu, ${ }^{1}$ Wei Xie, ${ }^{2}$ \\ and Yong Q. Zhang ${ }^{1}$ \\ ${ }^{1}$ Key Laboratory of Molecular and Developmental Biology, Institute of Genetics and Developmental Biology, Chinese Academy of Sciences, Beijing 100101, \\ China, and ${ }^{2}$ Key Laboratory for Developmental Genes and Human Disease, Ministry of Education, Institute of Life Sciences, Southeast University, Nanjing \\ 210096, China
}

The precise regulation of synaptic growth is critical for the proper formation and plasticity of functional neural circuits. Identification and characterization of factors that regulate synaptic growth and function have been under intensive investigation. Here we report that brain tumor (brat), which was identified as a translational repressor in multiple biological processes, plays a crucial role at Drosophila neuromuscular junction (NMJ) synapses. Immunohistochemical analysis demonstrated that brat mutants exhibited synaptic overgrowth characterized by excess satellite boutons at NMJ terminals, whereas electron microscopy revealed increased synaptic vesicle size but reduced density at active zones compared with wild-types. Spontaneous miniature excitatory junctional potential amplitudes were larger and evoked quantal content was lower at brat mutant NMJs. In agreement with the morphological and physiological phenotypes, loss of Brat resulted in reduced FM1-43 uptake at the NMJ terminals, indicating that brat regulates synaptic endocytosis. Genetic analysis revealed that the actions of Brat at synapses are mediated through mothers against decapentaplegic (Mad), the signal transduction effector of the bone morphogenetic protein (BMP) signaling pathway. Furthermore, biochemical analyses showed upregulated levels of Mad protein but normal mRNA levels in the larval brains of brat mutants, suggesting that Brat suppresses Mad translation. Consistently, knockdown of brat by RNA interference in Drosophila S2 cells also increased Mad protein level. These results together reveal an important and previously unidentified role for Brat in synaptic development and endocytosis mediated by suppression of BMP signaling.

\section{Introduction}

The synapse is a specialized intercellular junction devoted to communication between neurons and their targets. Proper growth and regulation of synapses are critical to the normal neuronal function. The Drosophila neuromuscular junction (NMJ) is an effective model system to dissect molecular mechanisms of synaptic development. Multifarious factors and molecular signaling pathways, such as actin regulators, endocytic proteins,

Received Jan. 25, 2013; revised June 16, 2013; accepted June 19, 2013.

Author contributions: W.S., Y.C., and Y.Q.Z. designed research; W.S., Y.C., G.G., and Q.W. performed research; W.S., Y.C., D.W., J.R., Z.X., and W.X. contributed unpublished reagents/analytic tools; W.S., Y.C., Z.X., W.X., and Y.Q.Z. analyzed data; W.S. and Y.Q.Z. wrote the paper.

This work was supported by grants from the National Science Foundation of China (NSFC; 31171041) and the Ministry of Science and Technology of China (MOST; 2012CB517903) to W.X., The Strategic Priority Research Program of the Chinese Academy of Sciences XDA01010105 to Z.X., and finally the Strategic Priority Research Program $B$ of the Chinese Academy of Sciences (KSCX2-EW-R-05 and XDB02020400) and the NSFC (30930033 and 30871388) to Y.Q.Z. We thank J. Knoblich, R. Wharton, M. O'Connor, C. Goodman, V. Budnik, D. Frank, S. J. Newfeld, E. M. De Robertis, P. ten Dijke, S. Sigrist, and A. DiAntonio for various antibodies, mutants, and transgenic flies. We thank the Bloomington Stock Center and the Vienna Drosophila RNAi Center for fly stocks, the Developmental Studies Hybridoma Bank, University of lowa, for antibodies. Dr L. Yang at the EM facility of our institute assisted in the ultrastructural analysis of NMJ terminals. We thank members of the Zhang laboratory for discussions and our colleagues Drs Mei Ding, Xun Huang, Paul Lasko, Thomas Schwarz, and Hugo Bellen for comments and critical reading of the paper.

${ }^{*}$ W.S. and Y.C contributed equally to this work.

The authors declare no competing financial interests.

Correspondence should be addressed to Dr Yong Q. Zhang, Institute of Genetics and Developmental Biology, Chinese Academy of Sciences, Datun Road, Chao Yang District, Beijing 100101, China. E-mail: yqzhang@genetics.ac.cn.

DOI:10.1523/JNEUROSCI.0386-13.2013

Copyright $(\odot) 2013$ the authors $\quad 0270-6474 / 13 / 3312352-12 \$ 15.00 / 0$ ubiquitin-mediated protein degradation, bone morphogenetic protein (BMP), and wingless (Wnt) pathways play important roles at Drosophila NMJ synapses (Collins and DiAntonio, 2007; O'Connor-Giles et al., 2008; Giagtzoglou et al., 2009; Ball et al., 2010; Bayat et al., 2011).

BMP signaling is a major retrograde growth-promoting pathway at Drosophila NMJ synapses (Collins and DiAntonio, 2007; O'Connor-Giles et al., 2008; Ball et al., 2010; Bayat et al., 2011). The retrograde BMP signaling cascade is initiated by release of the ligand Glass bottom boat (Gbb) from the postsynaptic muscle and subsequent binding to the presynaptic type II BMP receptor wishful thinking (Wit). Upon ligand binding, Wit forms a complex with the type I receptors thickvein ( Tkv) and saxophone (Sax), resulting in their phosphorylation. In turn, phosphorylated type I receptors phosphorylate the Smad family transcriptional factor mothers against decapentaplegic (Mad). Mad is a signal transduction effector that, when phosphorylated, translocates to the nucleus of motoneurons to regulate transcription of target genes that control NMJ growth (Collins and DiAntonio, 2007; Ball et al., 2010; Bayat et al., 2011).

Brain tumor (Brat) contains multiple protein-protein interaction domains and is conserved throughout evolution from Caenorhabditis elegans to humans (Arama et al., 2000). Brat acts as a translational repressor in multiple developmental contexts through distinct mechanisms. During early embryogenesis, Brat forms a complex with the RNA-binding proteins Pumilio (Pum) and Nanos (Nos) and the RNA 5' cap-binding protein d4EHP 
(the Drosophila homolog of eIF4E) to suppress the translation of the morphogen Hunchback in the posterior (Sonoda and Wharton, 2001; Edwards et al., 2003; Cho et al., 2006). In the female germline, Brat acts together with Pum to repress the expression of Mad and the growth regulator $\mathrm{dMyc}$ to promote germline differentiation (Harris et al., 2011). During larval neurogenesis, Brat controls neuroblast self-renewal and neuronal differentiation (Bello et al., 2006; Betschinger et al., 2006; Lee et al., 2006; Stefanatos and Vidal, 2011). In the postmitotic neurons, Brat interacts with Pum and Nos to translationally repress the voltage-gated sodium channel subunit paralytic (para) and thereby modulate the excitability of motor neurons (Muraro et al., 2008). Pum and Nos regulate NMJ synapse development (Menon et al., 2004, 2009), but a possible role for Brat at synapses has not been demonstrated.

We report here that the NMJ terminals of brat mutants exhibit more numerous satellite boutons than do wild-type and that these mutant NMJs have reduced neurotransmission efficiency and defective endocytosis. Furthermore, our data indicate that Brat regulates synapse development and endocytosis by suppressing translation of the BMP signaling component Mad. Thus, our study unravels a novel role for brat at the NMJ and offers new insight into the regulation of BMP signaling for NMJ growth.

\section{Materials and Methods}

Drosophila stocks and genetics. Flies of either sex were cultured in standard cornmeal media at $25^{\circ} \mathrm{C}$ unless otherwise indicated. The $w^{1118}$ strain was used as the wild-type control. Other fly strains used were motor neuronspecific Ok6-Gal4 from M. O'Connor (University of Minnesota, Saint Paul, $\mathrm{MN}$ ) and muscle-specific Mhc-Gal4 from C. Goodman (Howard Hughes Medical Institute, Chevy Chase, MD). The mutant $b r a t^{11}$ strain was from D. Frank (Washington University, St. Louis, MO; Frank et al., 2002), both $\mathrm{brat}^{150}$ and $\mathrm{brat}^{192}$ were from J. Knoblich (Institute of Molecular Biotechnology, Vienna, Austria; Betschinger et al., 2006), UAS-brat ${ }^{R N A i}$ was from the Vienna Drosophila RNAi Center (No. V31333), and UAS-brat was from R. Wharton (The Ohio State University, Columbus, OH; Sonoda and Wharton, 2001). The Mad mutant mad $^{12}$ was from S. J. Newfeld (Arizona State University, Tempe, AZ; Takaesu et al., 2005). A mad RNAi transgenic line was from E. M. De Robertis (Howard Hughes Medical Institute, Los Angeles, CA; Eivers et al., 2009). The remaining strains, da-Gal4, elav-Gal4, $w i t^{A 12}, t k v^{7}, d a d^{J 1 E 4}$, and $D f(2 L) B S C 162$, were obtained from the Bloomington Stock Center. For rescue experiments, meiotic recombination (OK6Gal4, brat ${ }^{11} / \mathrm{CyO}-G F P$ ) and interchromosomal combinations (elav-Gal4; $\mathrm{brat}^{11} / \mathrm{CyO}-\mathrm{GFP}, \mathrm{brat}^{11} / \mathrm{CyO}-\mathrm{GFP} ; \mathrm{Mhc}-$ Gal4/TM6B, and $\mathrm{brat}^{192} / \mathrm{CyO}-$ GFP; UAS-flag-brat/TM6B) were constructed according to conventional procedures. For elav-Gal4 rescue, only female progeny were collected for analyses. The identities of the recombinants as parental stocks were verified by phenotypic and immunochemical analyses. Nonbalancer progeny were selected to examine rescue effects.

Immunohistochemical analyses. Dissections and immunohistochemical analyses of wandering third-instar larvae of either sex were performed as described previously (Jin et al., 2009; Yao et al., 2011). For immunostaining of Brp and glutamate receptors, larvae were dissected in a normal medium (128 mM NaCl, $2 \mathrm{~mm} \mathrm{KCl,} 4 \mathrm{~mm} \mathrm{MgCl}_{2}, 35.5 \mathrm{~mm}$ sucrose, and 5 mM HEPES, pH 7.3; Jan and Jan, 1976) supplemented with $2 \mathrm{~mm}$ L-glutamate and fixed in ice-cold methanol for $5 \mathrm{~min}$. For immunostaining of other proteins, late third-instar larvae were dissected in $\mathrm{Ca}^{2+}$-free standard saline and fixed in 4\% paraformaldehyde for 30-60 min. The following monoclonal antibodies were obtained from the Developmental Studies Hybridoma Bank: anti-CSP(cysteine-string protein; 6D6; 1:500), anti-Bruchpilot (nc82; 1:50), anti-GluRIIA (1:50), and anti-Discs large (Dlg; 4F3; 1:1000). Other antibodies used included rabbit antiphosphorylated Mad (1:500) from P. ten Dijke (Leiden University, Leiden, the Netherlands; Persson et al., 1998), rabbit anti-glutamate receptor IID (GluRIID; 1:2500) from S. Sigrist (Free University Berlin, Berlin, Germany; Qin et al., 2005), rabbit GluRIIB from (1:2500) from A.
DiAntonio (Washington University, WA), anti-Flag (Sigma-Aldrich No. F3165; 1:2000), and FITC- and Texas red-conjugated anti-horseradish peroxidase (HRP; 1:200) from Jackson ImmunoResearch. The corresponding secondary antibodies, goat anti-mouse and goat anti-rabbit IgGs labeled with Alexa Fluor 488 or 568 (Invitrogen), were used at 1:1000. Motor neuron nuclei were labeled with TO-PRO-3 iodide (Invitrogen No. T3605; 1:2000). All images were acquired with a Leica SP5 confocal microscope and processed using Adobe Photoshop 8.0.

For quantification of bouton number, images of NMJ 4 stained with anti-CSP were analyzed with NIH ImageJ as described previously (Jin et al., 2009; Yao et al., 2011). Satellite boutons were defined as the small boutons emanating from the NMJ branch or from larger parental boutons (Dickman et al., 2006; O'Connor-Giles et al., 2008). For quantification of pMad level at NMJs, staining intensities were measured within the HRP-positive NMJ 4 in abdominal segments A2 and A3. For quantification of pMad level in motor neuron nuclei, staining intensities normalized to the nuclei dye signals were quantified with ImageJ software.

Western analysis and S2 cell culture. Western blotting analysis of larval brains and Schneider 2 (S2) cells were conducted as previously described (Jin et al., 2009; Wang et al., 2010) using the following primary antibodies: anti-Flag (Sigma No. F3165; 1:20000), anti-pMad (Cell Signaling Technology No. 9516; 1:1000), anti-Mad (Santa Cruz Biotechnology No. 15810; 1:500), and anti-actin (Millipore Bioscience Research Reagents No. mAb1501; 1:100,000). The secondary HRP-labeled antibodies were obtained from Sigma-Aldrich. Protein bands were visualized with an ECL kit from Millipore. S2 cells were cultured in Sf-900 II serum-free medium (Invitrogen No. 10902). A brat dsRNA, produced according to Harris et al. (2011), was transfected using Cellfectin II (Invitrogen No. 10362) according to the manufacturer's instructions. Cells were harvested for Western analysis $2 \mathrm{~d}$ after transfection.

Transgenic constructs and anti-brat antibodies. A UAS-Flag-Brat construct was generated using the Drosophila Gateway Vector system. The Brat coding sequence was amplified from a cDNA clone (DGRC LD16270) by PCR and recombined into the pCR8/GW/TOPO vector (Invitrogen No. k250020). After the in vitro recombination reaction between the entry clone and a destination vector (DGRC, pTFMW), the UAS-flag-brat expression clone was generated. Brat polyclonal antibodies were raised in rats against a His-tagged fusion protein bearing amino acid residues $723-1037$ of Brat. For immunostaining, the antibody was used at a 1:400 dilution. Brat monoclonal antibody $3 \mathrm{~A} 9$ was generated in mouse using a His fusion protein bearing Brat amino acid residues 367 767 and a GST fusion protein bearing Brat amino acid residues 723-1037 at a ratio of 1:1. The 3A9 antibody recognized both peptides of 367-767 and $723-1037$ aa as indicated by an ELISA assay. For Western analysis, 3A9 was used at a 1:1000 dilution.

Quantification of Mad mRNA level. Total RNA was isolated from thirdinstar larval brains of the various genotypes using Trizol Reagent (Invitrogen No. 15596) according to the manufacturer's instructions. Total RNA was reverse transcribed into single-stranded cDNA using SuperScript III First-Strand Synthesis System (Invitrogen No. 18080). Quantitative PCR was performed using the Agilent Mx3000p real-time PCR detection system and the QuantiTect SYBR Green PCR kit (Qiagen No. 204141). The primers for detecting mad mRNA were as follows: $5^{\prime}$-AA TCCGTGGTGGTAGTTGCAG-3' and 5'-AACAACTCCGTGATCGTT GAC-3'. The primers 5' -GCTGAGCGTGAAATCGTCCGTG-3' and 5' CCCAAGAACGAGGGCTGGAACA- $3^{\prime}$ were used to detect actin mRNA. The expression level of mad mRNA was normalized to that of actin mRNA. At least three biological repeats were performed for statistical analysis.

FM1-43 uptake assay. For the FM1-43 dye loading assay, we followed previously published protocols (Verstreken et al., 2008; Wang et al., 2010). Late third-instar larvae were dissected in the normal medium $(128 \mathrm{~mm} \mathrm{NaCl}$, $2 \mathrm{~mm} \mathrm{KCl}, 4 \mathrm{~mm} \mathrm{MgCl}$, $35.5 \mathrm{~mm}$ sucrose, and 5 mM HEPES, pH 7.3; Jan and Jan, 1976), then washed with $1.5 \mathrm{mM} \mathrm{Ca}^{2+}$ normal medium for $30 \mathrm{~s}$. Motor axons innervating muscles were gently cut without disturbing the underlying musculature to eliminate electrical firing from the CNS. To load the fluorescent FM1-43 dye (Invitrogen No. T-35356) into boutons, preparations were incubated for $5 \mathrm{~min}$ in high- $\mathrm{K}^{+}(90 \mathrm{mM})$ saline containing $10 \mu \mathrm{M}$ FM1-43 (Invitrogen), and then vigorously washed three times for $5 \mathrm{~min}$ per wash in $\mathrm{Ca}^{2+}$-free saline. For the rescue experiments, flies were cultured at $18^{\circ} \mathrm{C}$ 
starting from the embryo stage and the FM1-43 uptake assay was also performed at $18^{\circ} \mathrm{C}$. The loaded synapses were imaged on a Leica SP 5 confocal microscope using a $40 \times$ water-immersion lens.

Electron microscopy and morphometric anal$y$ sis. Larval tissue sections for EM analysis were prepared according to procedures described previously (Liu et al., 2010; Liu et al., 2011). Wandering third-instar larvae were dissected in HL-3 saline (128 mm NaCI, $2 \mathrm{~mm} \mathrm{KCl,} 4 \mathrm{~mm}$ $\mathrm{MgCl}_{2}, 35 \mathrm{~mm}$ sucrose, 5 mm HEPES, $\mathrm{pH}$ 7.4) and fixed at $4^{\circ} \mathrm{C}$ overnight in a mixture of $2 \%$ glutaraldehyde and $2 \%$ formaldehyde in $0.1 \mathrm{M}$ sodium cacodylate buffer, $\mathrm{pH} 7.4$, followed by several rinses with cacodylate buffer. Right and left hemi-segments from abdominal segment A2 or A3 were separated from the larval fillets and postfixed for $2 \mathrm{~h}$ with $1 \% \mathrm{OsO}_{4}$ in cacodylate buffer. The preparations were stained en bloc for $1 \mathrm{~h}$ with saturated uranyl acetate in $50 \%$ ethanol before dehydration in a graded series of ethanol solutions. The samples were embedded in Spurr resin (Sigma-Aldrich). Serial longitudinal ultrathin sections $(70 \mathrm{~nm}$ thick) of NMJ 6/7 were prepared on a Leica UC6 ultramicrotome using a diamond knife. Grids were poststained with saturated uranyl acetate in $50 \%$ ethanol and $1 \%$ lead citrate $(\mathrm{pH}$ 12) and examined under a Jeol JEM-1230 electron microscope. Images of sections through the midline of type $1 \mathrm{~b}$ boutons were captured with a Ganton820 digital CCD camera. For quantification of the size and density of synaptic vesicles (SVs) at active zones, images of $>20$ individual boutons from at least four animals of each genotype were analyzed. The diameters and the number of SVs within a $200 \mathrm{~nm}$ radius of the transmitter release site (T-bar) were measured using ImageJ.

Electrophysiology. Excitatory junctional potentials (EJPs) and spontaneous miniature EJPs (mEJPs) at NMJs were recorded using intracellular electrodes (Jin et al., 2009; Wang et al., 2010). Wandering third-instar larvae were dissected in $\mathrm{Ca}^{2+}$-free HL3.1 saline. The gut and fat were removed and the body wall was spread open to expose the nerves and muscles. Intracellular microelectrodes were pulled from borosilicate glass (World Precision Instruments) on a glass puller (P-2000, Sutter Instrument) and filled with $3 \mathrm{M} \mathrm{KCl}$. Electrodes with resistances of $10-20 \mathrm{M} \Omega$ were used for the experiments. Recordings were performed at $18^{\circ} \mathrm{C}$ with an Axoclamp $2 \mathrm{~B}$ amplifier (Molecular Devices) in Bridge mode. Data were digitized with a Digidata $1322 \mathrm{~A}$ digitizer (Molecular Devices) and acquisition was controlled by Pclamp 9.1 (Molecular Devices). Both EJP and mEJPs were recorded in HL3.1 saline containing $0.23 \mathrm{mM} \mathrm{Ca}^{2+}$. For EJP recordings, a Grass S48 stimulator with SIU-5 isolator (AstroGrass) coupled to a suction electrode was used to stimulate the nerve with $0.3 \mathrm{~Hz}$ suprathreshold pulses. A total of 25-30 EJPs were recorded from NMJ 6 of abdominal segment A3 for each animal, followed by mEJP recording for $120 \mathrm{~s}$. Only recordings from muscles with resting membrane potentials more polarized than $50 \mathrm{mV}$ and input resistances $>6 \mathrm{M} \Omega$ were analyzed. All data were
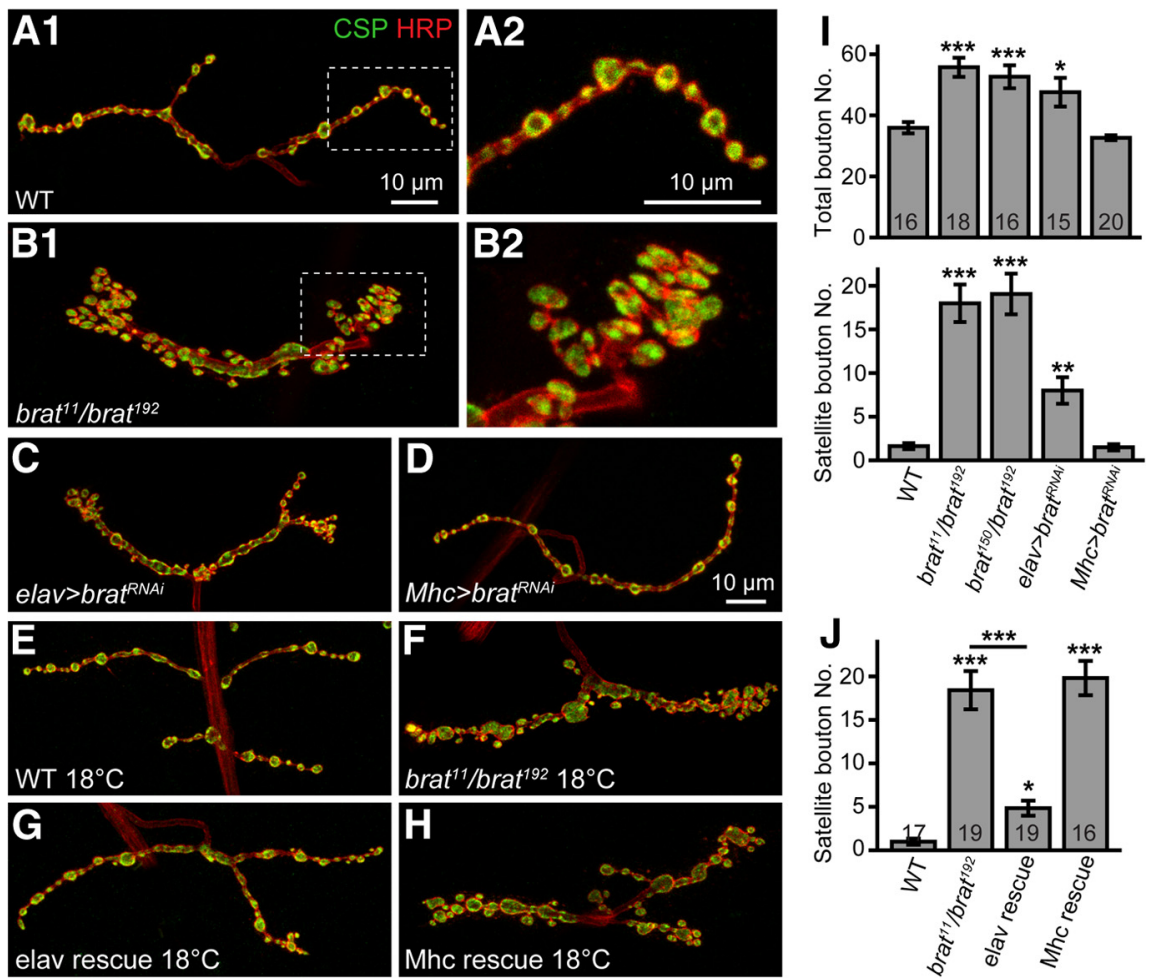

Figure 1. brat mutants have more numerous satellite boutons. All images are projections of confocal z-stacks of NMJ 4 synapses from abdominal segment A2 or A3 of a third-instar larva double-labeled with anti-CSP (green) and anti-HRP (red). $A-D$, brat mutants and flies with presynaptic but not postsynaptic RNAi knockdown of brat showed excess satellite boutons instead of the typical boutons arranged in a "beads-on-a-chain" pattern in wild-type. $\boldsymbol{A 2}, \boldsymbol{B 2}$, Boxed areas in $\boldsymbol{A} \mathbf{1}$ and $\mathbf{B 1}$, respectively, at higher magnification. The genotypes are wild-type $(\boldsymbol{A})$, brat $^{11} /$ brat $^{192}(\boldsymbol{B})$, elav-Gal4/UAS-brat ${ }^{\text {RNAi }}(\boldsymbol{C})$, and UAS-brat ${ }^{R N A i} /+;$ Mhc-Gal4/+ (D). E-H, The increased number of satellite boutons in brat mutants was rescued by presynaptic but not postsynaptic expression of brat at $18^{\circ} \mathrm{C}$. The genotypes are wild-type $(\boldsymbol{E}), b r a t^{11} / b_{r a t} t^{192}(\boldsymbol{F})$, and presynaptic $(\boldsymbol{G})$ and postsynaptic $(\boldsymbol{H})$ expression of brat on a brat mutant background $\left(\mathbf{G}\right.$, elav-Gal4; brat $^{11} /$ brat $^{192}$; UAS-flag-brat/+ and $\boldsymbol{H}$, brat ${ }^{11} /$ brat $^{192}$; Mhc-Gal4/UAS-flag-brat). Scale bar, $10 \mu \mathrm{m}$. I, J, Bar graphs showing statistical results of total and satellite bouton number at $25^{\circ} \mathrm{C}(\boldsymbol{I})$ and satellite bouton number at $18^{\circ} \mathrm{C}(\boldsymbol{J})$ of different genotypes. The number of animals analyzed for each genotype is indicated in the column. Statistical significance was calculated using one-way ANOVA $\left({ }^{*} p<0.05 ;{ }^{* *} p<0.01 ;{ }^{* *} p<0.001\right.$; error bars denote SEM).
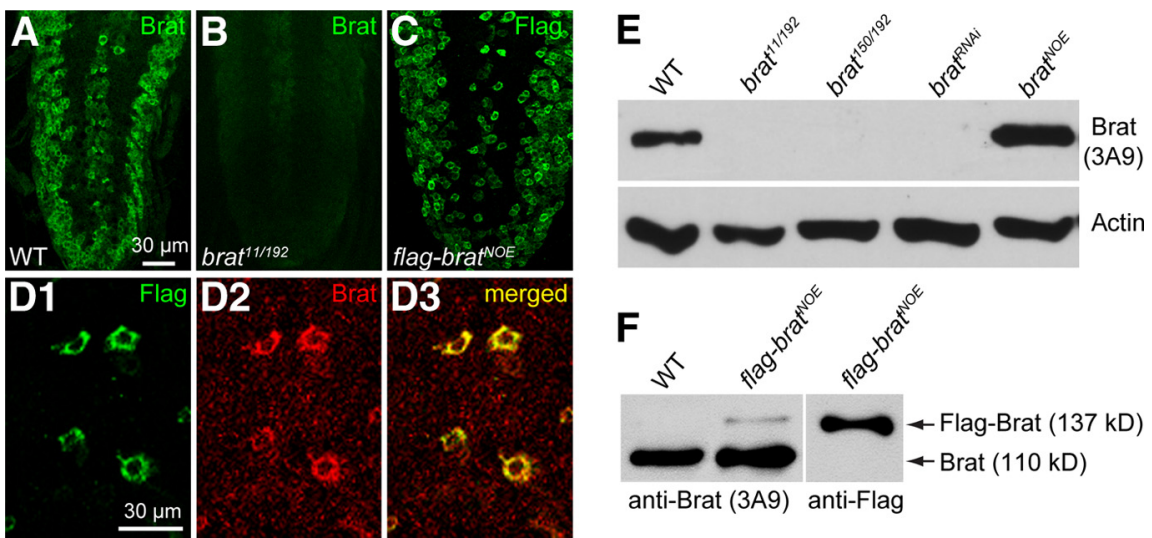

Figure 2. Characterization of loss- and gain-of-function mutants of brat by immunochemical analysis. $\boldsymbol{A}, \boldsymbol{B}$, The ventral nerve cord (VNC) of third-instar larvae of wild-type $(\boldsymbol{A})$ and brat $^{11} /$ brat $^{192}$ mutants $(\boldsymbol{B})$ was stained with a rat anti-Brat serum. C, Anti-Flag staining of VNC-expressing Flag-Brat pan-neuronally under the control of elav-Gal4 (elav-Gal4/+; UAS-flag-brat/+). D1-D3, Enriched expression of Flag-Brat in the soma of motoneurons in the VNC costained with anti-Flag (D1, green) and anti-Brat (D2, red). Flag-Brat was overexpressed in motor neurons driven by OK6-Gal4 in brat mutant background (OK6-Gal4/+, brat $^{11} /$ brat $^{192}$; UAS-flag-brat/+). $\boldsymbol{E}$, Western analysis of larval brains with a monoclonal anti-Brat antibody 3A9. The genotypes are as follows: wild-type, brat $^{11} /$ brat $^{192}$, brat $^{150} / \mathrm{brat}^{192}$, UAS-brat $^{\text {RNAi }} /+$; da-Gal4/+, and elav-gal4/+; UAS-brat/+. F, Identification of UAS-flag-brat transgenic lines (elav-Gal4/+; UAS-flagbrat/+) by Western blotting using anti-Flag and the monoclonal anti-Brat $3 \mathrm{~A} 9$. 

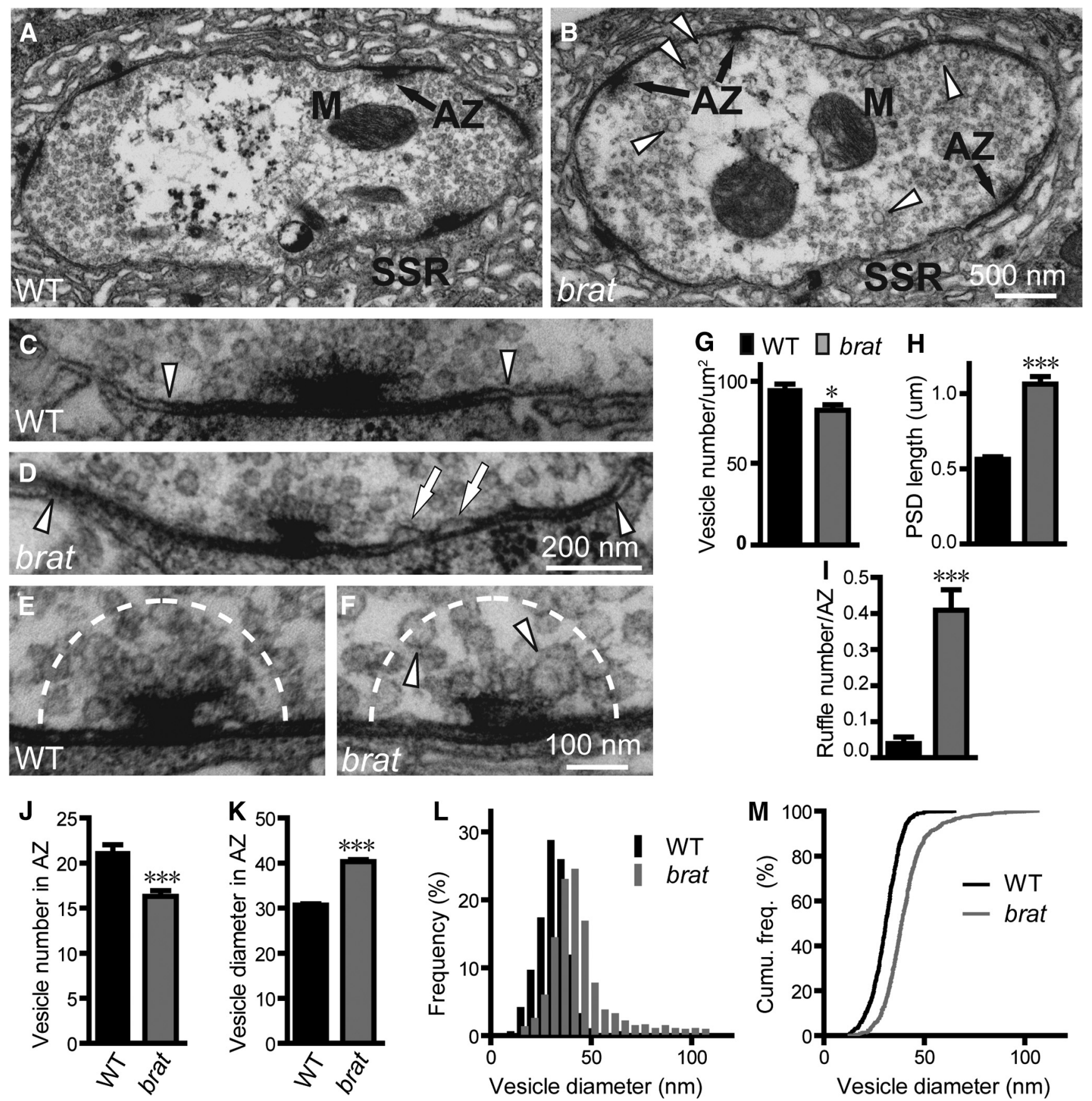

Figure 3. brat mutant boutons have fewer but larger SVs at active zones. Micrographs of synaptic boutons from wild-type $(A, C, E)$ and brat ${ }^{11} / b r a t^{192}$ mutants $(B, D, F)$ are shown. Mitochondria (M), AZs, and SSR are indicated in $\boldsymbol{A}$ and $\boldsymbol{B}$. $\boldsymbol{C} \boldsymbol{F}$ show active zones with SVs clustered around a T-bar at higher magnification. Arrowheads in $\boldsymbol{C}$ and $\boldsymbol{D}$ indicate electron-dense membranes; arrows in $\boldsymbol{D}$ denote presynaptic membrane ruffles. Arrowheads in $\boldsymbol{F}$ indicate enlarged vesicles around T-bar. Scale bars: $\boldsymbol{B}, 500 \mathrm{~nm} ; \boldsymbol{D}, 200 \mathrm{~nm} ;$ and $\boldsymbol{F}, 100 \mathrm{~nm}$. $\boldsymbol{G}$, Bar graphs showing statistical results of mean vesicle number per square micrometer of cross-sectioned boutons. $\boldsymbol{H}, \boldsymbol{I}$, Bar graphs showing statistical results of mean PSD length $(\boldsymbol{H})$ and the number of presynaptic membrane ruffles per AZ $(\boldsymbol{I})$. $\boldsymbol{J}, \boldsymbol{K}$, Bar graphs showing statistical analyses of the number and diameter of SVs within a $200 \mathrm{~nm}$ radius of active zones demarcated by dashed lines in $\boldsymbol{E}$ and $\boldsymbol{F}$. $\boldsymbol{L}, \boldsymbol{M}$ The frequency distribution and cumulative probability plot of vesicle diameters in the defined area of AZs ( $n=676$ for wild-type and $n=735$ for mutants from $\geq 4$ animals). Statistical significance was calculated using Student's $t$ test $\left({ }^{*} p<0.05 ;{ }^{* * *} p<0.001\right.$; error bars denote SEM).

analyzed with Clampfit 9.1 software. Quantal content was calculated by dividing the mean EJP amplitude corrected for nonlinear summation by the mean $\mathrm{mEJP}$ amplitude with a reversal potential of $0 \mathrm{mV}$ according to Martin's equation (Martin, 1955).

Statistical analyses. All data are expressed as mean \pm SEM. Statistical comparisons were performed using SPSS 18.0. For multiple comparisons among the different genotypes in Figures 1 and 5-9, one-way ANOVAs with post hoc multiple pairwise comparisons were performed. For pairwise comparisons between wild-type Drosophila and mutants in Figures
3 and 4 , two-tailed Student's $t$ tests were performed $\left({ }^{*} p<0.05,{ }^{* *} p<\right.$ 0.01 , and ${ }^{* * *} p<0.001$; error bars indicate SEM).

\section{Results}

brat regulates synaptic growth

To characterize the role of Brat in synaptic development and function, we first examined NMJ morphology in brat mutants (Fig. 1). We examined three strong or null alleles brat ${ }^{11}$, brat $^{150}$, 
and brat $^{192}$, all nonsense mutations from independent sources (Arama et al., 2000; Frank et al., 2002; Betschinger et al., 2006). These mutant alleles showed no Brat expression by immunostaining and Western blotting (Fig. 2A,B,E). Compared with the wild-type, both $\mathrm{brat}^{11} /$ brat $^{192}$ and brat $^{150} /$ brat $^{192}$ mutants exhibited more boutons at muscle 4 NMJ (NMJ 4) on segments A2 and A3. The mean total bouton number in brat $^{11} /$ brat $^{192}$ mutants was $55.72 \pm 3.13$ (mean \pm SEM), a $55 \%$ increase over the wild-type (35.94 $\pm 1.86 ; p<0.001$; Fig. $1 \mathrm{~A}, B, I)$, whereas $\mathrm{brat}^{150} / \mathrm{brat}^{192}$ mutants exhibited a similar $46.4 \%$ increase compared with wild-type $(52.63 \pm 3.76, p<$ 0.001 ; Fig. $1 I)$. The number of satellite boutons emanating from the main branch or primary boutons (Dickman et al., 2006; O'Connor-Giles et al., 2008) was significantly higher in mutants $(18.00 \pm 2.15$ for brat $^{11} /$ brat $^{192}$ mutants, $19.06 \pm 2.33$ for brat $^{150} /$ brat $^{192}$ mutants, $1.63 \pm 0.34$ for wild-typs; $p<0.001$ compared with wild-type for both mutants; Fig. 1I). To eliminate the possibility that this supernumerary satellite bouton phenotype was caused by a background mutation on the brat $^{192}$ chromosome, we also examined the NMJ phenotype of brat ${ }^{192}$ hemizygous mutants (brat $\left.t^{192} / D f(2 L) p r-A 16\right)$ and found that they also exhibited excess satellite boutons (data not shown). The excess satellite boutons largely account for the increase in the total bouton number in brat mutants.

To confirm that the excess satellite bouton phenotype was caused specifically by brat mutations, we examined synaptic terminals in animals where Brat was knocked down by RNA interference driven by the ubiquitous da-Gal4. Western analysis confirmed effective Brat knockdown (Fig. 2E). As expected, Brat knockdown recapitulated the supernumerary satellite boutons of brat mutants (data not shown). Tissue-specific RNAi knockdown was then used to examine whether Brat functions presynaptically or postsynaptically. Targeted RNAi knockdown in presynaptic neurons under control of the pan-neuronal elav-Gal4 induced an NMJ phenotype similar to that of $\mathrm{brat}^{11} / \mathrm{brat}^{192}$ mutants (Fig. $1 B, C, I)$. In contrast, postsynaptic knockdown of Brat by the muscle-specific Mhc-Gal4 did not alter NMJ morphology (Fig. $1 A, D, I)$.

To further verify that the distinct NMJ phenotype in brat mutants was due to loss of Brat function, we performed tissuespecific rescue experiments using the UAS-Gal4 system (Brand and Perrimon, 1993). We generated a UAS-flag-brat transgenic line (Fig. 2C,D,F). We observed no obvious rescue of the overgrown phenotype of brat mutants when the crosses of UAS-flagbrat driven by elav-Gal4 or Mhc-Gal4 were cultured at $25^{\circ} \mathrm{C}$, probably due to an excess Flag-Brat protein. Indeed, a slightly higher level of Brat than the endogenous level (Fig. $2 F$ ) could give rise to abnormal NMJs (data not shown), indicating that NMJ growth is sensitive to Brat protein levels. As the Gal4 protein exhibits lower activity at $18^{\circ} \mathrm{C}$ than that at $25^{\circ} \mathrm{C}$ (Wucherpfennig et al., 2003; Greenspan, 2004; Harris et al., 2011), we performed denote SEM).
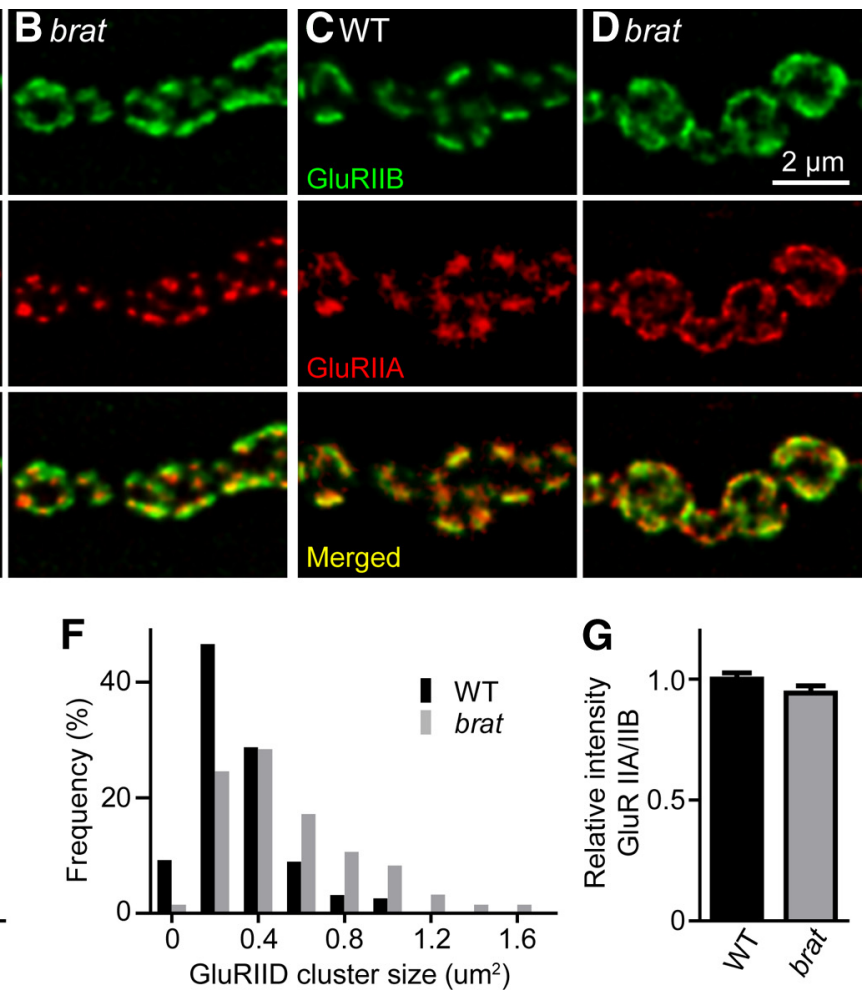

Figure 4. The cluster size of glutamate receptors is increased in brat mutants. $A-D$, Confocal images of larval NMJ synapses from brat $^{11} /$ brat $^{192}$ mutants ( $n \geq 15$ NMJs). Statistical significance was calculated using Student's $t$ test $\left(^{* * *} p<0.001\right.$; error bars

the rescue experiments again at $18^{\circ} \mathrm{C}$ and found that the satellite bouton number in $\mathrm{brat}^{11} / \mathrm{brat}^{192}$ mutants $(18.42 \pm 2.19)$ was significantly reduced by the presynaptic expression of brat driven by elav-Gal4 $(4.84 \pm 0.87 ; p<0.001$; Fig. $1 J)$, though not restored to the wild-type level. Motor neuron-specific expression of brat driven by Ok6-Gal4 showed rescue effects (3.31 $\pm 1.01 ; p<$ 0.001 ) similar to that by elav-Gal4. In contrast, postsynaptic expression of brat driven by Mhc-Gal4 did not rescue the NMJ deficit $(p>0.05$; Fig. $1 J)$. Neuronal expression of Brat via an independent UAS-brat insertion (Sonoda and Wharton, 2001; we confirmed in Fig. $2 E$ ) produced rescue effects similar to that of UAS-flag-brat (data not shown). These tissue-specific RNAi knockdown and rescue experiments demonstrate that Brat regulates NMJ development primarily on the presynaptic side.

\section{Synaptic ultrastructure is altered in brat mutants}

In addition to light microscopic analyses, we also examined NMJ synapses of brat mutants at the ultrastructural level. Presynaptic structures essential for neurotransmitter release at NMJ terminals include mitochondria, SVs, and active zones with T-bars, although the most prominent postsynaptic structure is the subsynaptic reticulum (SSR) composed of a meshwork of convoluted muscle plasma membranes (Fig. 3A). The presynaptic mitochondria and postsynaptic SSR appeared largely normal in brat $^{11} /$ brat $^{192}$ mutants (Fig. 3, compare $B$ with $A$ ). However, the vesicle density within the whole bouton was moderately but significantly reduced $\left(94.71 \pm 3.50 / \mu \mathrm{m}^{2}\right.$ in WT vs $82.24 \pm 3.31$ / $\mu \mathrm{m}^{2}$ in mutants, $p<0.05$; Fig. $\left.3 G\right)$, although a small subpopulation of vesicles (Fig. $3 B$, arrowheads) were larger in brat $^{11} /$ brat $^{192}$ mutants compared with the wild-type. There were 


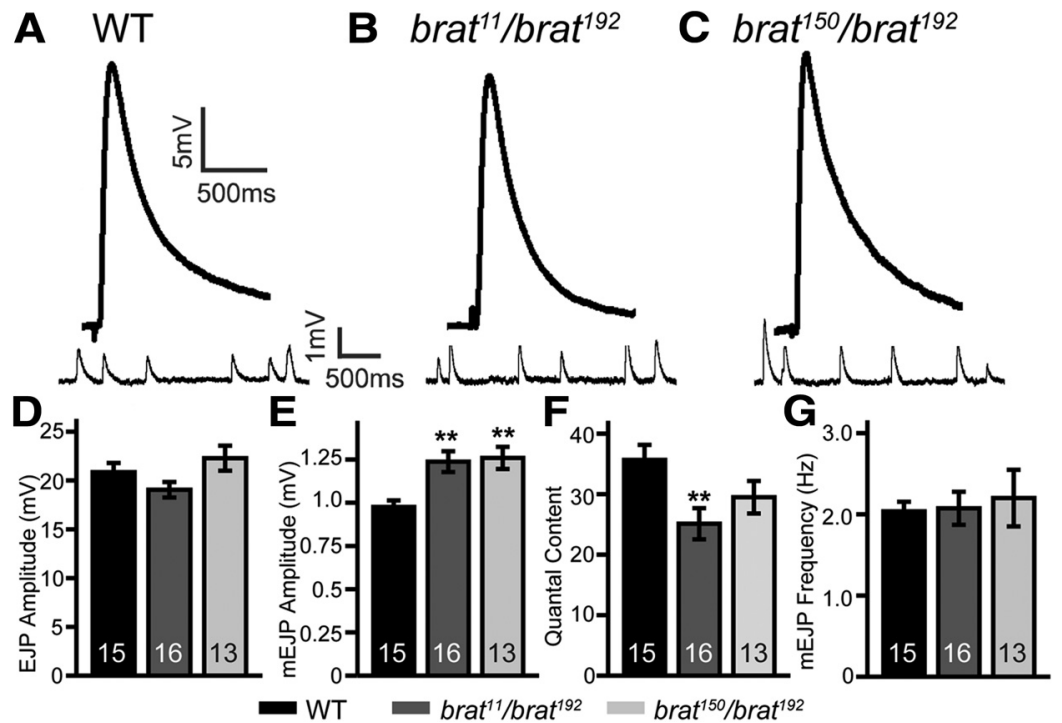

Figure 5. Neurotransmission efficacy is decreased in brat mutants. $A-C$, Representative EJP and mEJP traces of wild-type, brat $^{17} /$ brat $^{192}$ and brat $^{150} /$ brat $^{192}$ mutants. D-G, Bar graphs showing the statistical results of average EJP amplitude (D), mEJP amplitude $(\boldsymbol{E})$, quantal content $(\boldsymbol{F})$, and $\mathrm{mEJP}$ frequency $(\boldsymbol{G})$ of different genotypes. The number of animals analyzed for each genotype is indicated in each column. Statistical significance was calculated using one-way ANOVA $\left({ }^{* *} p<0.01\right.$; error bars denote SEM).
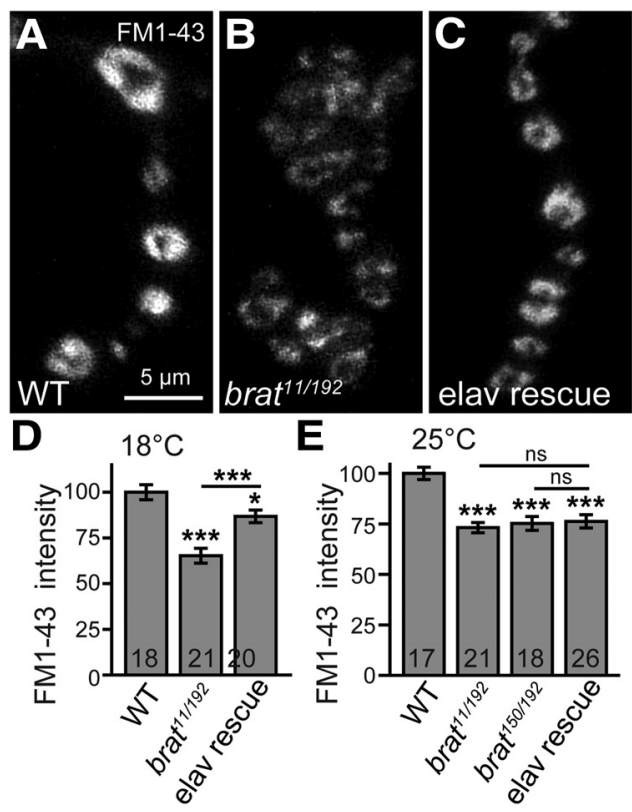

Figure 6. Brat is required for the normal FM1-43 uptake at NMJ terminals. $A-E$, FM1-43 uptake results performed at $18^{\circ} \mathrm{C}(\boldsymbol{A}-\boldsymbol{D})$ and $25^{\circ} \mathrm{C}(\boldsymbol{E})$. The wild-type synapses were labeled brightly by endocytosed FM1-43 at $18^{\circ} \mathrm{C}(\boldsymbol{A})$. In contrast, brat $^{11} /$ brat $^{192}$ mutants $(\boldsymbol{B})$ showed a significant reduction in the fluorescent intensity of endocytosed FM1-43. The reduced FM1-43 intensity in brat mutants was partially rescued by presynaptic expression of brat at $18^{\circ} \mathrm{C}(\mathrm{C}$, elav-Gal4/+; brat $^{11} /$ brat $^{192}$; UAS-flag-brat/+). Scale bar, $5 \mu \mathrm{m}$. D, E, Statistical results of relative intensities of loaded FM1-43 dye in different genotypes at $18^{\circ} \mathrm{C}(\boldsymbol{D})$ and $25^{\circ} \mathrm{C}(\boldsymbol{E})$. Statistical significance was calculated using one-way ANOVA $\left({ }^{*} p<0.05\right.$; ${ }^{* *} p<0.01 ;{ }^{* * *} p<$ 0.001; error bars denote SEM).

also fewer synaptic vesicles within a $200 \mathrm{~nm}$ radius of the T-bar in brat $^{11} /$ brat $^{192}$ mutants $(16.31 \pm 0.62)$ than wild-type $(21.13 \pm$ $0.91 ; p<0.001$; Fig. $3 E, F, J)$. The mean SV diameter within the area of active zones (AZs) was significantly larger in $\mathrm{brat}^{11} / \mathrm{brat}^{192}$ mutants compared with wild-type $(40.33 \pm 0.43 \mathrm{~nm}$ vs $30.67 \pm 0.28 \mathrm{~nm} ; p<$ 0.001 ; Fig. $3 E, F, K)$. A histogram and cumulative probability plot showed that $91 \%$ of wild-type SVs were $<40 \mathrm{~nm}$, compared with only $55 \%$ of SVs in brat $^{11}$ / brat $^{192}$ mutants (Fig. $3 L, M$ ). Hence, loss of Brat reduced vesicle density but resulted in a subpopulation of larger vesicles at NMJ terminals, a phenotype similar to that observed in many endocytic mutants, such as AP180/lap, dap160, and tweek (Zhang et al., 1998; Koh et al., 2004; Verstreken et al., 2009). The larger SVs may contain more glutamate neurotransmitter, consistent with the greater amplitudes of spontaneous $\mathrm{mEJP}$ in brat mutants (Fig. 5; see below). We also observed a significant increase in the number of presynaptic membrane ruffles within the electron-dense membranes at the $\mathrm{AZ}$ in brat mutants $(0.41 \pm 0.06$ ruffles per $\mathrm{AZ}$ for $b r a t^{11} / b_{r a t}{ }^{192}$ mutants vs $0.04 \pm 0.02$ ruffles per AZ for the wild-type; Fig. 3I), suggesting a defect in endocytosis, cell adhesion, or both.

The mean length of the postsynaptic density (PSD; Fig. 3C,D, arrowheads) where glutamate receptors are enriched was longer in brat mutants compared with wild-type $(1.06 \pm 0.05 \mu \mathrm{m}$ vs $0.56 \pm 0.02 \mu \mathrm{m} ; p<0.001$; Fig. $3 H)$, consistent with immunostaining results showing an enlarged cluster size of GluRIID, an obligatory subunit of functional receptors (Featherstone et al., 2005; Qin et al., 2005), in brat mutants $\left(0.32 \pm 0.13 \mu \mathrm{m}^{2}\right.$ in WT vs $0.58 \pm 0.25 \mu \mathrm{m}^{2}$ in brat $^{11} /$ brat $^{192}$ mutants, $p<0.001$; Fig. $4 A, B, E, F)$. We observed similar enlarged cluster size of GluR subunits IIA and IIB (Fig. $4 C, D$ ), but the ratio of GluRIIA to GluRIIB intensities was normal in brat mutants (Fig. 4G).

brat mutants show increased quantal size but decreased neurotransmission efficacy at NMJ terminals

To examine the functional consequences of these altered NMJ synapses in brat mutants, we recorded EJPs and spontaneous mEJPs at NMJ 6/7 using intracellular electrodes. In $0.23 \mathrm{~mm}$ $\mathrm{Ca}^{2+}$ HL3.1 saline, neither mean EJP amplitude $(19.06 \pm 0.79$ $\mathrm{mV}$ for brat $^{11} / \mathrm{brat}^{192}, 22.29 \pm 1.28 \mathrm{mV}$ for $\mathrm{brat}^{150} / \mathrm{brat}^{192} \mathrm{vs}$ $20.86 \pm 0.94 \mathrm{mV}$ for wild-type; Fig. $5 A-D$ ) nor mEJP frequency $\left(2.07 \pm 0.20 \mathrm{~Hz}\right.$ for $\mathrm{brat}^{11} / \mathrm{brat}^{192}, 2.20 \pm 0.35 \mathrm{~Hz}$ for $\mathrm{brat}^{150} /$ brat $^{192}$ vs $2.04 \pm 0.12 \mathrm{~Hz}$ for wild-type; Fig. $\left.5 A-C, G\right)$ was significantly altered in brat mutants $(p>0.05)$. However, the mean mEJP amplitude was significantly larger in brat mutants $(1.24 \pm$ $0.06 \mathrm{mV}$ for $\mathrm{brat}^{11} / \mathrm{brat}^{192}, 1.26 \pm 0.06 \mathrm{mV}$ for $\mathrm{brat}^{150} / \mathrm{brat}^{192} \mathrm{vs}$ $0.98 \pm 0.04 \mathrm{mV}$ for wild-type; $p<0.01$ for both; Fig. $5 E$ ), consistent with the larger synaptic vesicles that presumably contain more glutamate in brat mutant boutons (Fig. 3). The enlarged GluR cluster size (Fig. 4) may also contribute to the increased mEJP amplitudes in brat mutants.

The number of vesicles released per stimulus (quantal content) is a measure of synaptic transmission efficacy and is calculated by dividing EJP amplitude (after correction for nonlinear summation) by mEJP amplitude. The estimated quantal content in heteroallelic brat $^{150} / \mathrm{brat}^{192}$ mutants was reduced, but not significantly different from wild-type ( $p>0.05$; Fig. $5 F)$, whereas that in brat $^{11} /$ brat $^{192}$ mutants was significantly lower compared 
with the wild-type $(25.10 \pm 2.58$ quanta/stimulus vs $36.28 \pm 2.60$ quanta/ stimulus for wild-type; $p<0.01$; Fig. $5 F)$. The weaker phenotype of the brat ${ }^{150} / \mathrm{brat}^{192}$ mutant is consistent with the molecular nature of the mutation; $\mathrm{brat}^{150}$ is a nonsense mutation resulting in the C-terminal 112 aa region deleted, whereas brat $^{11}$ has a larger 259 aa C-terminal deletion (Arama et al., 2000; Betschinger et al., 2006). These results show that brat mutants have a larger quantal size but decreased neurotransmission efficacy at NMJ synapses.

\section{Synaptic endocytosis is impaired in brat mutants}

In brat mutants, the NMJ terminals show excessive satellite boutons, reduced vesicle density but increased vesicle size at $\mathrm{AZs}$, increased mEJP amplitudes, and lower transmission efficiency (Figs. 1, 3-5). Similar synaptic defects were observed in many endocytic mutants, such as AP180, dap160, dynamin, and endophilin (Zhang et al., 1998; Verstreken et al., 2002; Koh et al., 2004; Marie et al., 2004; Dickman et al., 2006), we therefore examined whether brat regulates synaptic vesicle endocytosis using the lipophilic fluorescent dye FM1-43. At Drosophila NMJ terminals, FM1-43 binds to synaptic membranes, is internalized during endocytosis, and gets trapped in synaptic vesicles, where its fluorescence can be measured after washout of extracellular dye, providing a measure of endocytic efficiency (Verstreken et al., 2008; Wang et al., 2010). Dissected larvae were stimulated with $90 \mathrm{~mm} \mathrm{KCl}$ for $5 \mathrm{~min}$ in the presence of $10 \mu \mathrm{M}$ FM1-43 to induce transmitter release and concomitant endocytosis of the dye. After washing with $\mathrm{Ca}^{2+}$ free saline, the accumulated FM1-43 in live NMJ 4 synapses was imaged by confocal microscopy. Compared with wild-type NMJ boutons, brat mutant boutons exhibited significantly lower FM1-43 fluorescence at $25^{\circ} \mathrm{C}(73.2 \%$ and $75.2 \%$ of the wild-type fluorescence intensity in $\mathrm{brat}^{11} / \mathrm{brat}^{192}$ and brat $^{150} / \mathrm{brat}^{192}$ mutants, respectively; $p<$ 0.001 ; Fig. 6E). However, the reduced FM1-43 intensity in brat mutants was not rescued by the presynaptic expression of flag-brat at $25^{\circ} \mathrm{C}$ (Fig. $6 E$ ), probably due to an inappropriate protein level of Flag-Brat. We then cultured the crosses at $18^{\circ} \mathrm{C}$, which allowed for a lower Brat expression from elav-Gal4> UAS-flag-brat and better rescue for NMJ phenotypes (Fig. 1). Under these conditions, the relative intensity of FM1-43 fluorescence in $\mathrm{brat}^{11} / \mathrm{brat}^{192}$ mutants was $65.2 \%$ of wildtype intensity at $18^{\circ} \mathrm{C}(p<0.001$; Fig. $6 A, B, D)$ and was partially but significantly rescued to $86.77 \%$ of the wild-type by the presynaptic expression of brat $(p<0.001$; Fig. $6 C, D)$, indicating an endocytic defect at brat NMJ terminals. denote SEM).
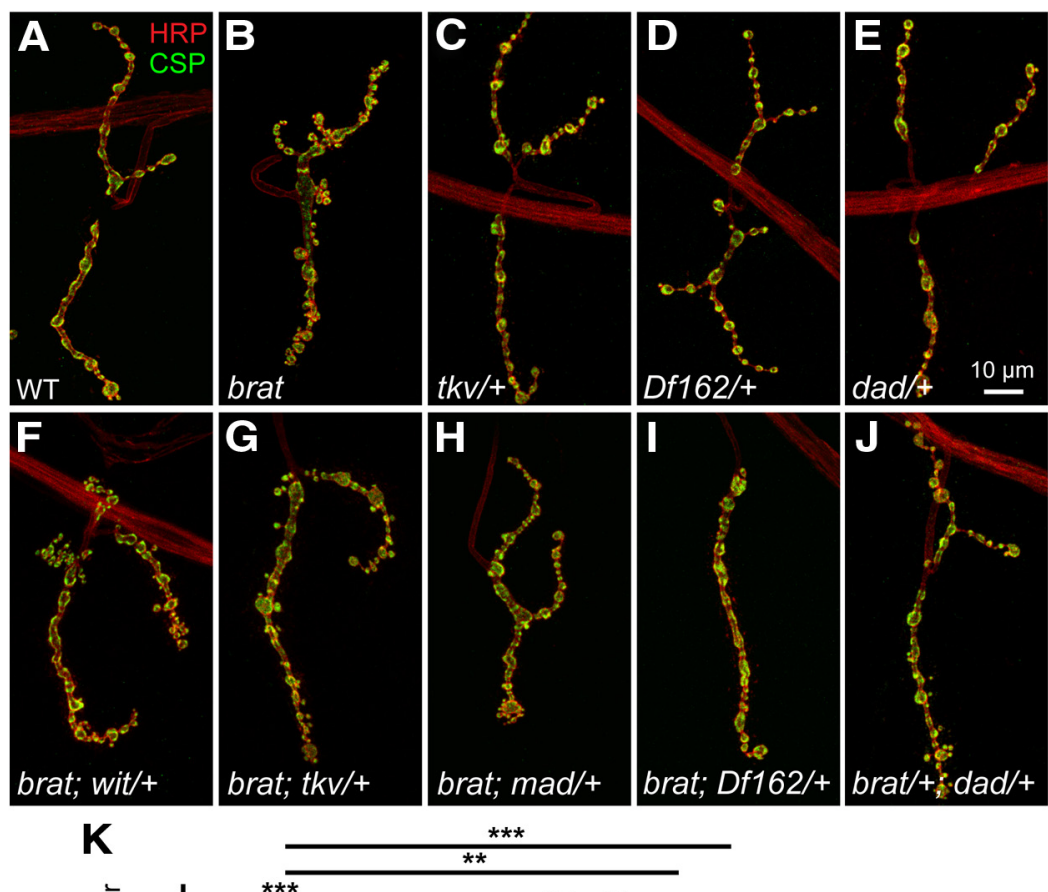

$* * *$
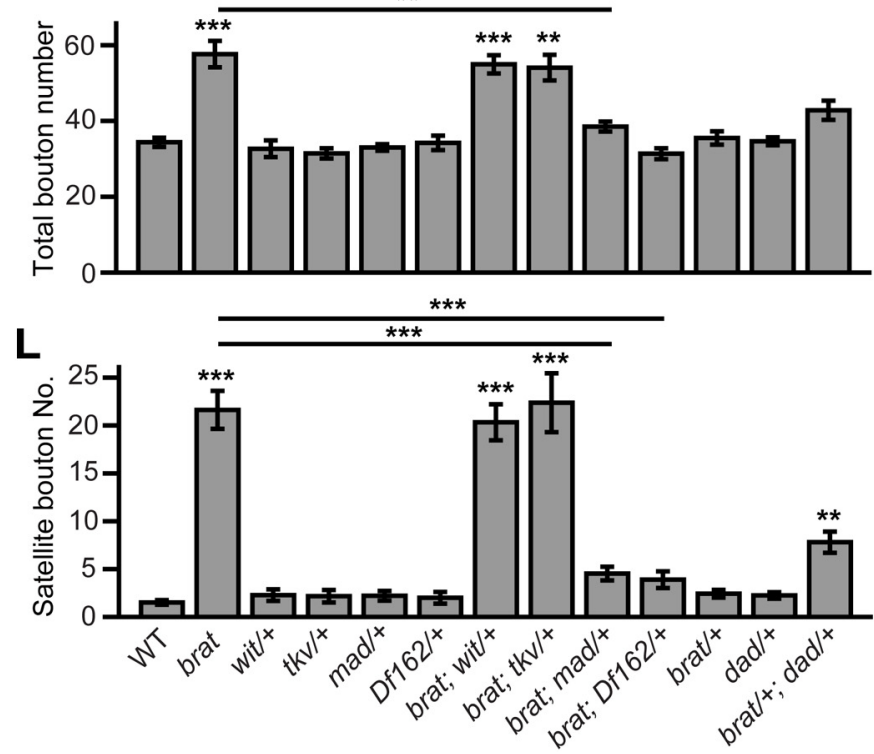

Figure 7. Synaptic overgrowth in brat mutants is rescued by decreasing the dose of mad. $\boldsymbol{A}-\boldsymbol{J}$, Confocal images of NMJ 4 double-labeled with anti-CSP (green) and anti-HRP (red). Wild-type (A), $\operatorname{brat}^{11} / \mathrm{brat}^{192}(\boldsymbol{B}), \mathrm{tkv}^{7} /+(\boldsymbol{C}), \mathrm{Df}(2 \mathrm{~L}) 162 /+(\boldsymbol{D})$, and $\operatorname{dad}^{\prime 1 E 4} /+(\boldsymbol{E})$ showed normal NMJ morphology. $\boldsymbol{F}, \boldsymbol{G}$, Removing one copy of wit or tkv did not suppress the synaptic overgrowth in

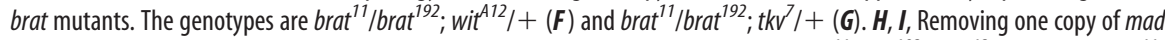
rescued synaptic overgrowth of brat mutants to the wild-type level. The genotypes are brat $^{11} / \mathrm{brat}^{192} ; \operatorname{mad}^{12} /+(\boldsymbol{H})$ and $b^{12 a t^{11} /}$ brat $^{192} ;$ Df(2L)162/ + (I). J, Trans-heterozygous brat ${ }^{192} /+;$ dad $^{11 E 4} /+$ mutants showed more satellite boutons compared with wild-type. Scale bar, $10 \mu \mathrm{m} . \boldsymbol{K}, \boldsymbol{L}$, Quantification of total bouton number $(\boldsymbol{K})$ and satellite bouton number $(\boldsymbol{L})$ of NMJ 4 for various

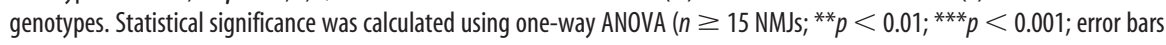

Synaptic defects of brat mutants are rescued by reducing the dose of $\mathrm{mad}$

Brat suppresses the translation of the BMP signaling molecule Mad during germline development (Harris et al., 2011) and enhanced BMP signaling leads to the development of excess satellite boutons at NMJs (Sweeney and Davis, 2002; Collins and DiAntonio, 2007; O'Connor-Giles et al., 2008; Zhao et al., 2013). These findings suggest that the effects of loss of Brat function at NMJ terminals may be mediated by BMP hyperactivation. We therefore examined synaptic overgrowth in brat mutants with reduced 


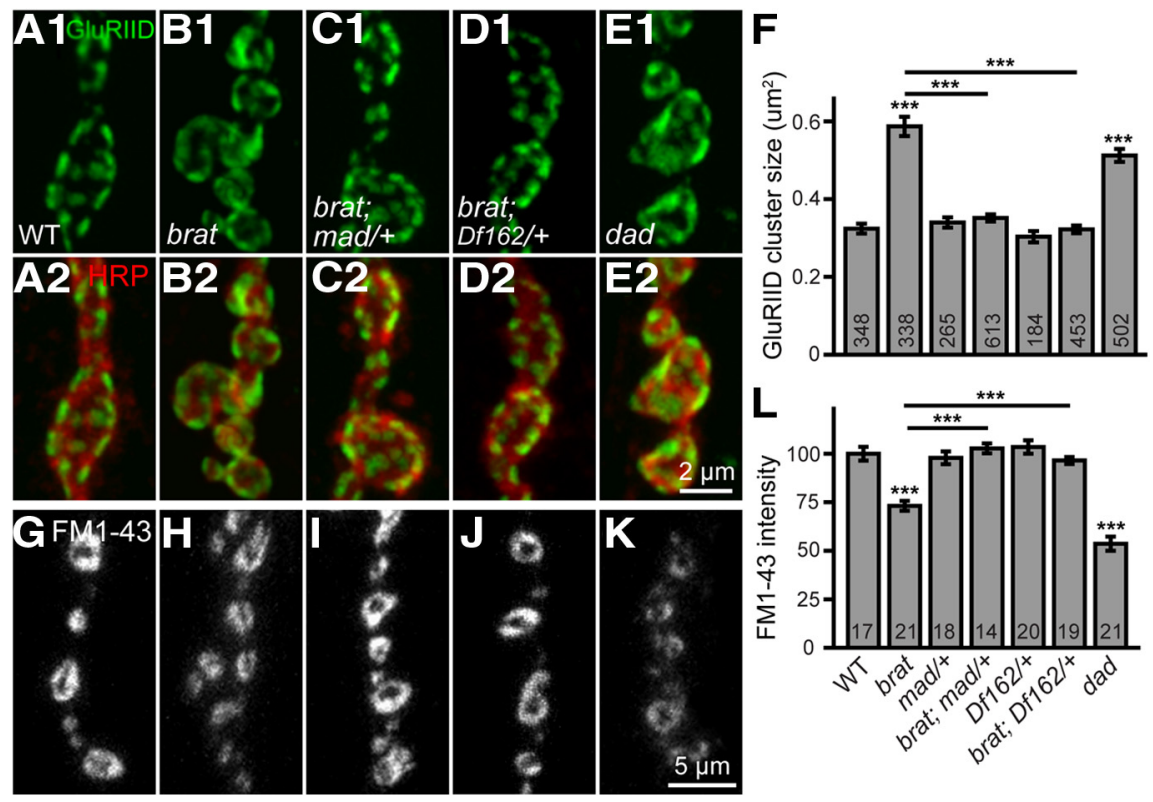

Figure 8. Enlarged GluRIID cluster size and defective endocytosis in brat mutants are rescued by reducing the dose of mad. $\boldsymbol{A}-\boldsymbol{E}$, Confocal images of NMJ 4 boutons double-labeled with anti-GluRIID (green) and anti-HRP (red). The enlarged GluRIID cluster size in brat $^{11} /$ brat $^{192}$ mutants $(\boldsymbol{B})$ was fully rescued by mad ${ }^{12} /+(\boldsymbol{C})$ and $D f(2 L) 162 /+(\boldsymbol{D})$ to wild-type. $\boldsymbol{E}$, A homozygous hypomorphic dad $^{\text {JIE4 }}$ also caused enlarged GluRIID cluster size. $\boldsymbol{F}$, Quantification of GluRIID cluster size of different genotypes. The number of GluRIID cluster analyzed for each genotype is indicated in the columns. $\mathbf{G}-\boldsymbol{K}$, FM1-43 uptake at NMJ 4 of different genotypes. The genotypes are $\mathbf{G}$, wild-type, $\boldsymbol{H}$, brat $^{11} / \mathrm{brat}^{192}, \boldsymbol{I}$, brat $^{11} / \mathrm{brat}^{192} ;$ mad $^{12} /+, \boldsymbol{J}$, brat $^{11} / \mathrm{brat}^{192} ; \mathrm{Df}(2 \mathrm{~L}) 162 /+$, and $\boldsymbol{K}$, dad $^{11 E 4}$. $\boldsymbol{L}$, Statistical results of relative intensities of loaded FM1-43 dye at NMJ boutons of different genotypes. Scale bars: $\boldsymbol{E} 1,2 \mu \mathrm{m} ; \boldsymbol{K}, 5$ $\mu \mathrm{m}$. The number of animals analyzed for each genotype is indicated in the columns. Statistical significance was calculated using one-way ANOVA (*** $p<0.001$; error bars denote SEM).

BMP signaling. Mutating one copy of wit (wit $\left.{ }^{A 12}\right)$ or $t k v\left(t k v^{7}\right)$ had no effect on NMJ growth and did not rescue the overgrowth of NMJs in brat mutants ( $p>0.05$; Fig. $7 C, F, G, K, L)$, suggesting that synaptic overgrowth in brat mutants is independent of the dose of the BMP receptors Wit or Tkv. However, removal of one copy of mad (heterozygous mad ${ }^{12}$ or deletion $D f(2 L) 162$ that uncovers $\mathrm{mad}$ ) had no effect on NMJ growth on the wild-type genetic background but significantly suppressed the excess bouton and satellite bouton phenotype of brat mutants (for satellite bouton, $4.53 \pm 0.71$ for $\mathrm{brat}^{11} / \mathrm{brat}^{192}$; $\mathrm{mad}^{12} /+, 3.90 \pm 0.87$ for brat $^{11} / \mathrm{brat}^{192} ; \mathrm{Df}(2 \mathrm{~L}) 162 /+$ vs $21.63 \pm 1.95$ for $\mathrm{brat}^{11} / \mathrm{brat}^{192}$; Fig. $7 D, H, I, K, L)$. Conversely, trans-heterozygotes of brat $^{192}$ and dad $^{\text {IIE4 }}$ (dad encodes an inhibitory Smad that negatively regulates BMP signaling) showed significantly more satellite boutons compared with wild-type, whereas the single heterozygous brat ${ }^{192}$ or dad $d^{I E 4}$ mutants showed normal NMJ morphology (Fig. 7E,J,L). Together, these results indicate that synaptic overgrowth in brat mutants may result from increased mad activity, consistent with Western results showing that Mad protein level was upregulated in brat mutants (see Fig. 9).

brat not only regulates NMJ development, but also affects the cluster size of glutamate receptors and endocytosis (Figs. 3, 4, 6). We therefore examined whether mad played a role in these bratregulated processes. Both brat and dad mutants exhibited larger postsynaptic clusters of GluRIID receptors and endocytosis defects. Reducing the dose of mad by half in heterozygous mad $^{12}$ or $D f(2 L) 162$ mutants reversed the enlarged GluRIID cluster size (Fig. $8 A-D, F)$ and the reduced FM1-43 dye uptake in $\mathrm{brat}^{11} / \mathrm{brat}^{192} \mathrm{mu}-$ tants to wild-type levels (Fig. 8G-J,L), suggesting that changes in the cluster size of glutamate receptors and reduced endocytosis in brat mutants are caused by increased mad expression. This notion was further supported by the fact that FM1-43 uptake was significantly reduced in homozygous hypomorphic $\mathrm{dad}^{I I E 4}$ mutants compared with wild-type (Fig. $8 G, K, L$ ).

Reducing mad expression was sufficient to reverse the principal aberrant NMJ phenotypes in brat mutants, including supernumerary satellite boutons, enlarged glutamate receptor clusters, and reduced endocytosis, indicating that the functions of brat in NMJ development are mediated through mad.

\section{Mad protein level is increased in brat mutants}

We then examined whether the protein level of Mad was altered in brat mutants. Mad is an effector of BMP signaling and the phosphorylated Mad (pMad) level serves as a molecular read out of BMP signaling at NMJ terminals (Wang et al., 2007; O'Connor-Giles et al., 2008). The pMad intensity normalized to HRP intensity was dramatically elevated in $\mathrm{brat}^{11} /$ brat $^{192}$ mutant NMJ synapses compared with the wild-type $(p<0.001$; Fig. $9 A, B, E)$. Tissue-specific rescue experiments using different Gal4 drivers showed that presynaptic expression of Flag-Brat driven by elav-Gal4 rescued the elevated pMad staining at brat $^{11} /$ brat $^{192}$ mutant NMJs concomitant with a reduction in satellite bouton number (Fig. 9A2-C2), whereas postsynaptic (muscular) expression of Flag-Brat under the control of Mhc-Gal4 did not significantly rescue brat mutant NMJ phenotypes (Fig. 9B,D,E). Consistent with the elevated pMad level at NMJs, pMad level was also upregulated in motor neuron nuclei of brat mutants (Fig. 9F-J).

Western analysis of larval brains demonstrated that the protein level of Mad in larval brains was higher in brat mutants and RNAi knockdown animals (4.24-fold increase for $\mathrm{brat}^{11} / \mathrm{brat}^{192}$, 4.41 -fold increase for $\mathrm{brat}^{150} / \mathrm{brat}^{192}$, and 2.18-fold increase for brat ${ }^{R N A i}$ compared with wild-type; Fig. $\left.9 K, M\right)$. The pMad protein level also had a similar increase in brat mutants (Fig. $9 K, M$ ). The specificities of the anti-pMad and anti-Mad antibodies were verified by the reduced Mad and pMad band intensities on Western blots when mad expression was knocked down by RNA interference (RNAi; Fig. 9K). We observed that increased expression of Mad protein also led to an elevated level of pMad on the wild-type background (data not shown). Knockdown of brat by RNAi in S2 cells also led to a twofold increased Mad and pMad proteins (Fig. $9 \mathrm{~K}, \mathrm{M}$ ). In contrast, quantitative PCR showed normal mad mRNA level in brat mutant brains, whereas a significant reduction in mad mRNA level was observed when mad RNAi was expressed under the control of the ubiquitous $d a$-Gal4 promoter (Fig. 9L). Elevated levels of pMad and Mad proteins with normal mad mRNA expression in brat mutants indicate that Brat likely inhibits Mad translation.

\section{Discussion}

\section{Brat regulates synapse growth}

Brain tumor (brat) was first identified as a tumor suppressor in Drosophila (Gateff, 1978) and it is now known that Brat acts together with translational repressors Pum and Pum-Nos complex in multiple developmental contexts. It is also known that 
Pum and Nos play critical but distinct roles in NMJ synapse development and function. Boutons are larger and fewer in pum mutants, whereas neuronal pum overexpression leads to smaller and more numerous boutons (Menon et al., 2004). Pum selectively binds to the 3 ' untranslated regions of Nanos mRNA, eIF-4E mRNA, and GluR IIA mRNA, and thereby suppresses translation of the encoded proteins at NMJ synapses (Menon et al., 2004, 2009). nanos mutants show more boutons, significantly decreased expression of GluR IIA, and increased expression of GluR IIB (Menon et al., 2009). Through a series of elegant genetic, immunohistochemical, and biochemical experiments, Menon et al. (2009) propose an intricate regulatory network among Pumilio, Nanos, and their targets at Drosophila NMJ synapses.

Unlike Pum and Nos, which are localized neuronal cell bodies and at NMJ terminals (Menon et al., 2004, 2009), we detected enriched expression of Brat in the neuronal soma (Fig. 2), but no endogenous Brat or ectopically expressed, functional Flag-Brat at NMJ synapses by immunostaining (data not shown). However, Olesnicky et al. (2012) reported that Brat is localized at presynaptic NMJ terminals. They also reported fewer boutons in brat mutants rather than excess satellite boutons as we observed (Fig. 1), possibly due to the different antibodies and quantification methods used. The conflicts between their study and the present study remain to be addressed. However, in agreement with their study, we also found that Brat regulated NMJ synapse development on the presynaptic side (Fig. 1).

In the present study, we found that brat mutants exhibit an NMJ phenotype distinct from that of pum and nos mutants, characterized by excess satellite boutons (Fig. 1), reduced transmitter release efficiency (Fig. 5), and defective endocytosis (Fig. 6). Satellite boutons are small boutons that protrude from synaptic branches or from a larger parent bouton. Mutations in genes involved in endocytosis (Dickman et al., 2006), TGF $\beta / B M P$ signaling (O'ConnorGiles et al., 2008; Nahm et al., 2010a,b), actin cytoskeleton dynamics (Coyle et al., 2004; Rodal et al., 2008; Ball et al., 2010; Nahm et al., 2010a,b), neuronal excitability (Lee and $\mathrm{Wu}, 2010$ ), and several other processes (Khodosh et al., 2006; Korolchuk et al., 2007; Yao et al., 2009; Schulte et al., 2010) all lead to prominent satellite boutons at NMJ terminals. Many of these processes and signaling pathways closely interact, thus accounting for the common satellite phenotype of many mutants. For example, endocytosis attenuates BMP signaling (O'Connor-Giles et al., 2008) and
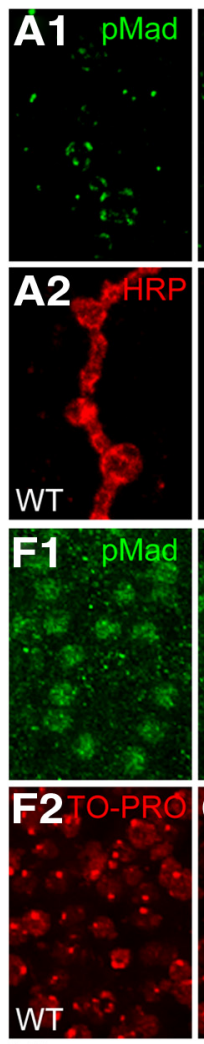

$\mathbf{M}$
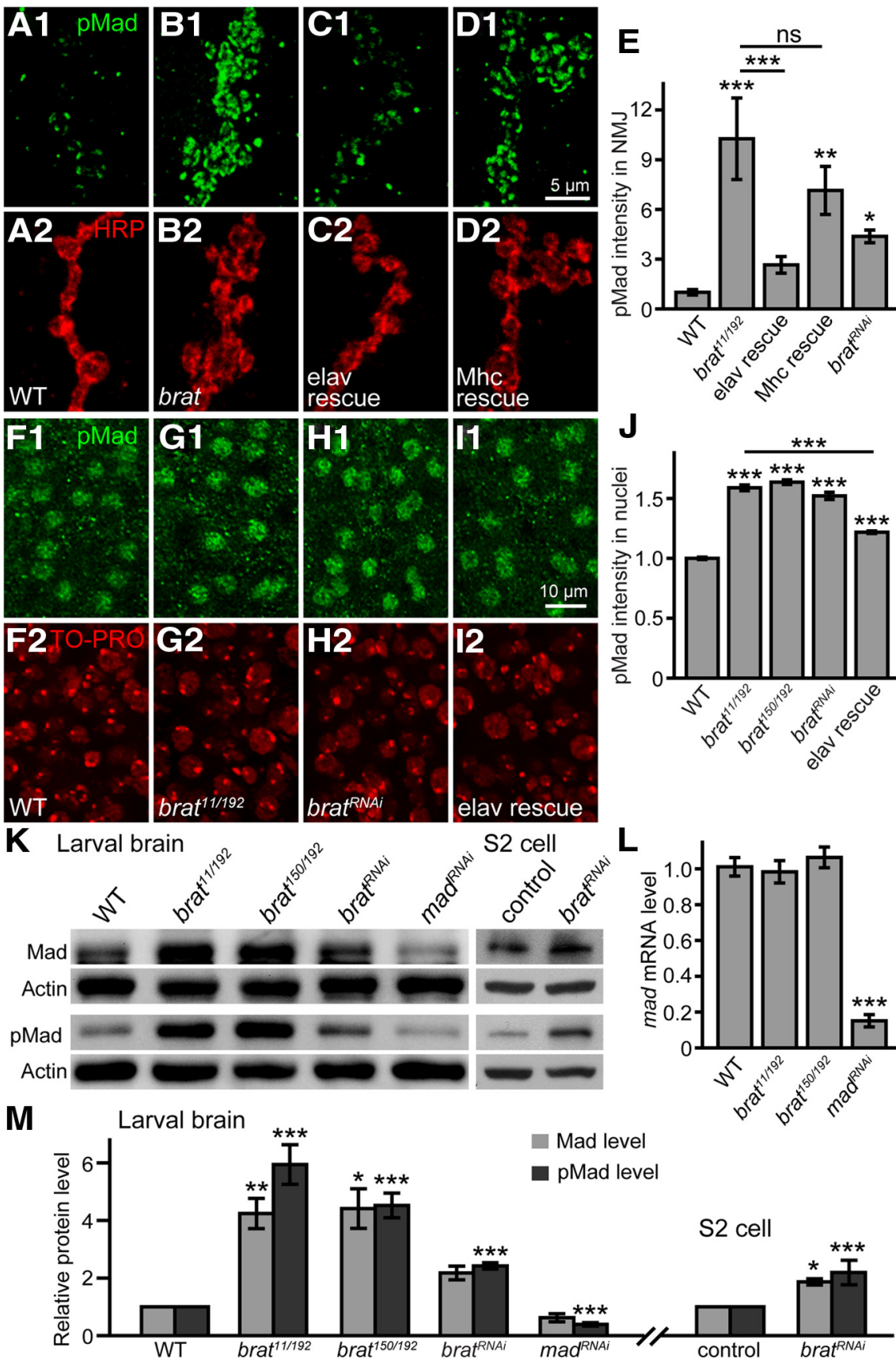

Figure 9. brat represses mad expression post-transcriptionally. $\boldsymbol{A}-\boldsymbol{D}$, Confocal images of NMJ 4 colabeled with anti-pMad (green) and anti-HRP (red). A, pMad staining in wild-type NMJ. $\boldsymbol{B}$, pMad level was dramatically upregulated in $\mathrm{brat}^{11} / \mathrm{brat}^{192}$ mutants compared with wild-type. $\boldsymbol{C}, \boldsymbol{D}$, The upregulated pMad level in brat mutants was partially rescued by neuronal $(\boldsymbol{C})$ but not muscular (D) expression of Flag-Brat. $\boldsymbol{E}$, Quantification of the intensity of pMad levels normalized to the anti-HRP staining intensity at NMJ terminals ( $n \geq 10 \mathrm{NMJs}$ ). $\boldsymbol{F}-\boldsymbol{I}$, Confocal images of motor neuron nuclei in third-instar larval VNCs double-stained with

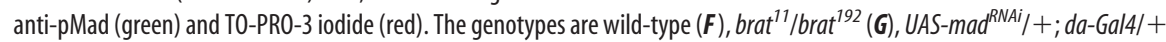
$(\boldsymbol{H})$, and elav-Gal4/+; rat $^{11} /$ brat $^{192} ;$ UAS-flag-brat/ $+(\boldsymbol{I})$. J, Quantification of pMad intensities in motor neuron nuclei of different genotypes ( $n \geq 100$ motor neurons from at least eight larvae). Scale bars: $\boldsymbol{D 1}, 5 \mu \mathrm{m} ; \boldsymbol{I 1}, 10 \mu \mathrm{m}$. $\boldsymbol{K}$, Western results of endogenous Mad and pMad proteins from larval brains of wild-type, $\operatorname{brat~}^{17} / \mathrm{brat}^{192}$, brat $^{150} / \mathrm{brat}^{192}$, and UAS-mad ${ }^{\mathrm{RNAi}} /+$; daGal4/ + and from $\$ 2$ cells treated with brat RNAi. L, Normalized levels of mad mRNAs determined using real-time PCR from larval brains of different genotypes. $M$, Statistical results of Mad and pMad protein levels in the larval brains of brat mutants and $S 2$ cells where brat was knocked down by RNAi ( $n \geq 5 ;{ }^{*} p<0.05 ;{ }^{* *} p<0.01$; ${ }^{* * *} p<0.001$; error bars denote SEM).

BMP signaling affects bouton formation via regulating actin dynamics by promoting the transcription of Trio, a Rac GTPase guanine exchange factor (Ball et al., 2010). As we discuss below, Brat may suppress satellite bouton formation by inhibiting BMP signaling. 


\section{Brat normally suppresses Mad expression to regulate synaptic growth}

Brat is a translational repressor containing no RNA-binding domains. During embryonic development, Brat suppresses mRNA translation by interacting with the RNA-binding proteins Pum and Nanos (Sonoda and Wharton, 2001; Cho et al., 2006). In the female germline, Brat acts with Pum to promote stem-cell differentiation by inhibiting target gene translation (Harris et al., 2011). In the nervous system, Brat is required for Pumilio-Nanos-dependent repression of the voltage-gated sodium channel subunit gene para in motoneurons, but dispensable for the negative regulation of para by the Pumilio-Nanos complex in other neuronal types (Muraro et al., 2008). It remains to be determined whether Brat interacts with Pumilio and Nanos to regulate genes involved in NMJ synapse growth. The distinct NMJ phenotypes between brat mutants and pum and nos mutants indicate that brat may regulate synapse development independently of pum and nos on the postsynaptic side. In support of their independent functions at postsynaptic NMJ synapses, brat mutants showed normal levels of GluRIIA and IIB (Fig. 4), whereas the level of GluRIIA and GluRIIB is upregulated in pum and nos mutant NMJ terminals, respectively (Menon et al., 2004, 2009). However, on the presynaptic side, our results showed that pMAD level was dramatically increased in pum $^{1}$ null mutants; trans-heterozygous pum $^{1} /+$; brat $^{192} /+$ mutants also showed an increased pMad level at NMJ synapses (data not shown). These results suggest that Mad might be repressed by the Pum-Brat complex in presynaptic neurons, consistent with a previous finding of inhibition of mad by the Pum-Brat complex in S2 cells (Harris et al., 2011).

The major NMJ phenotypes of brat mutants, including excess satellite boutons, increased GluR cluster size, and endocytic defects (Figs. 1, 4, 6), were rescued by decreasing the dose of the BMP signaling effector Mad (Figs. 7, 8). Both pMad and total Mad protein expression levels were higher, whereas mad mRNA expression was normal in the larval brains of brat mutants (Fig. 9). Furthermore, Brat knockdown by RNA interference in S2 cells also led to increased expression of Mad and pMad (Fig. 9). These results indicate that Brat normally acts to limit BMP signaling by suppressing the translation of Mad mRNA. In support of this conclusion, Brat specifically suppresses Mad translation via its $3^{\prime}$ untranslated region in S2 cells (Harris et al., 2011). The negative regulation of Mad by Brat in the nervous system is reminiscent of that described in ovarian stem-cell differentiation (Harris et al., 2011), suggesting that this is a conserved mechanism for BMP signaling regulation.

A significantly increased cluster size of glutamate receptors in brat mutants, as well as in dad mutants (Figs. 4, 8), indicating that the aberrantly elevated BMP signaling might lead to changes in postsynaptic receptor organization. Moreover, the enlarged GluR cluster size of brat mutants was completely reversed by heterozygous mad mutations (Fig. 8). How might Brat, which appears to act in presynaptic neurons, control postsynaptic GluR cluster size? Elevated BMP signaling may lead to abnormal F-actin dynamics at the presynaptic terminals via enhanced expression of Trio (Ball et al., 2010), but it is unknown whether altered presynaptic F-actin could contribute to the increased GluR cluster size on the postsynaptic side. However, two trans-synaptic complexes, neuroligin 1-neurexin and teneurins, have been reported to restrict GluR cluster size (Pielage et al., 2006; Li et al., 2007; Banovic et al., 2010;
Mosca et al., 2012). Furthermore, both neuroligin and teneurins are required to maintain the postsynaptic spectrin cytoskeleton (Mosca et al., 2012). Thus, it is conceivable that BMP signaling, negatively regulated by Brat through Mad, may affect one or both of these trans-synaptic signaling pathways. Alternatively, an as yet unidentified Brat target may regulate the postsynaptic cytoskeleton and the postsynaptic architecture.

\section{Brat regulates synaptic endocytosis by suppressing BMP signaling}

We provide multiple lines of independent evidence supporting that brat regulates synaptic endocytosis. First, brat mutants show excess satellite boutons, which are a general feature of endocytic mutants, such as dap160, endophilin, and eps15 (Koh et al., 2004, 2007; Dickman et al., 2006). Second, the NMJ boutons in brat mutants exhibited other abnormal characteristics of endocytic mutants, including larger but fewer vesicles (Fig. 3) and a concomitant increase in mEJP amplitudes (Fig. 5). Third, as with tweek, dap160, and twf mutants with defective endocytosis (Koh et al., 2004; Verstreken et al., 2009; Wang et al., 2010), the FM1-43 dye uptake was reduced at brat mutant NMJ (Fig. 6).

brat mutations led to endocytic defects as well as increased BMP signaling. Increased BMP signaling leads to excess satellite boutons (O'Connor-Giles et al., 2008), but it has not been known whether upregulation of BMP signaling results in endocytic defects. We show here that the endocytic defect in brat mutants could be rescued by reducing the dose of mad. Moreover, multiple mutations in $\mathrm{dad}$, a negative regulator of BMP signaling, resulted in excess satellite boutons and reduced FM1-43 dye uptake at NMJ terminals (Fig. 8; O'Connor-Giles et al., 2008). Thus, increased BMP signaling can result in endocytic defects, probably by affecting actin cytoskeleton, as Trio, a Rac GTPase guanine exchange factor, is a direct target of Mad (Ball et al., 2010). As endocytic defects lead to increased BMP signaling (O'Connor-Giles et al., 2008), there seems a mutual negative regulation between endocytosis and BMP signaling, i.e., a positive feedback loop for BMP signaling, at NMJ terminals.

In summary, our results provide genetic and biochemical evidence for a model in which Brat regulates synaptic growth and endocytosis at NMJ terminals by suppressing the translation of Mad, an effector of BMP signaling. This study describes a previously unknown regulatory mechanism for BMP signaling in the nervous system.

\section{References}

Arama E, Dickman D, Kimchie Z, Shearn A, Lev Z (2000) Mutations in the beta-propeller domain of the Drosophila brain tumor (brat) protein induce neoplasm in the larval brain. Oncogene 19:3706-3716. CrossRef Medline

Ball RW, Warren-Paquin M, Tsurudome K, Liao EH, Elazzouzi F, Cavanagh C, An BS, Wang TT, White JH, Haghighi AP (2010) Retrograde BMP signaling controls synaptic growth at the NMJ by regulating trio expression in motor neurons. Neuron 66:536-549. CrossRef Medline

Banovic D, Khorramshahi O, Owald D, Wichmann C, Riedt T, Fouquet W, Tian R, Sigrist SJ, Aberle H (2010) Drosophila neuroligin 1 promotes growth and postsynaptic differentiation at glutamatergic neuromuscular junctions. Neuron 66:724-738. CrossRef Medline

Bayat V, Jaiswal M, Bellen HJ (2011) The BMP signaling pathway at the Drosophila neuromuscular junction and its links to neurodegenerative diseases. Curr Opin Neurobiol 21:182-188. CrossRef Medline

Bello B, Reichert H, Hirth F (2006) The brain tumor gene negatively regulates neural progenitor cell proliferation in the larval central brain of Drosophila. Development 133:2639-2648. CrossRef Medline 
Betschinger J, Mechtler K, Knoblich JA (2006) Asymmetric segregation of the tumor suppressor brat regulates self-renewal in Drosophila neural stem cells. Cell 124:1241-1253. CrossRef Medline

Brand AH, Perrimon N (1993) Targeted gene expression as a means of altering cell fates and generating dominant phenotypes. Development 118: 401-415. Medline

Cho PF, Gamberi C, Cho-Park YA, Cho-Park IB, Lasko P, Sonenberg N (2006) Cap-dependent translational inhibition establishes two opposing morphogen gradients in Drosophila embryos. Curr Biol 16: 2035-2041. CrossRef Medline

Collins CA, DiAntonio A (2007) Synaptic development: insights from Drosophila. Curr Opin Neurobiol 17:35-42. CrossRef Medline

Coyle IP, Koh YH, Lee WC, Slind J, Fergestad T, Littleton JT, Ganetzky B (2004) Nervous wreck, an SH3 adaptor protein that interacts with Wsp, regulates synaptic growth in Drosophila. Neuron 41:521-534. CrossRef Medline

Dickman DK, Lu Z, Meinertzhagen IA, Schwarz TL (2006) Altered synaptic development and active zone spacing in endocytosis mutants. Curr Biol 16:591-598. CrossRef Medline

Edwards TA, Wilkinson BD, Wharton RP, Aggarwal AK (2003) Model of the brain tumor-pumilio translation repressor complex. Genes Dev 17: 2508-2513. CrossRef Medline

Eivers E, Fuentealba LC, Sander V, Clemens JC, Hartnett L, De Robertis EM (2009) Mad is required for wingless signaling in wing development and segment patterning in Drosophila. PloS One 4:e6543. CrossRef Medline

Featherstone DE, Rushton E, Rohrbough J, Liebl F, Karr J, Sheng Q, Rodesch CK, Broadie K (2005) An essential Drosophila glutamate receptor subunit that functions in both central neuropil and neuromuscular junction. J Neurosci 25:3199-3208. CrossRef Medline

Frank DJ, Edgar BA, Roth MB (2002) The Drosophila melanogaster gene brain tumor negatively regulates cell growth and ribosomal RNA synthesis. Development 129:399-407. Medline

Gateff E (1978) Malignant neoplasms of genetic origin in Drosophila melanogaster. Science 200:1448-1459. CrossRef Medline

Giagtzoglou N, Ly CV, Bellen HJ (2009) Cell adhesion, the backbone of the synapse: "vertebrate" and "invertebrate" perspectives. Cold Spring Harb Perspect Biol 1:a003079. CrossRef Medline

Greenspan RJ (2004) Fly pushing: the theory and practice of Drosophila genetics. Cold Spring Harbor, NY: CSHL.

Harris RE, Pargett M, Sutcliffe C, Umulis D, Ashe HL (2011) Brat promotes stem cell differentiation via control of a bistable switch that restricts BMP signaling. Dev Cell 20:72-83. CrossRef Medline

Jan LY, Jan YN (1976) Properties of the larval neuromuscular junction in Drosophila melanogaster. J Physiol 262:189-214. Medline

Jin S, Pan L, Liu Z, Wang Q, Xu Z, Zhang YQ (2009) Drosophila Tubulinspecific chaperone $E$ functions at neuromuscular synapses and is required for microtubule network formation. Development 136:1571-1581. CrossRef Medline

Khodosh R, Augsburger A, Schwarz TL, Garrity PA (2006) Bchs, a BEACH domain protein, antagonizes Rab11 in synapse morphogenesis and other developmental events. Development 133:4655-4665. CrossRef Medline

Koh TW, Verstreken P, Bellen HJ (2004) Dap160/intersectin acts as a stabilizing scaffold required for synaptic development and vesicle endocytosis. Neuron 43:193-205. CrossRef Medline

Koh TW, Korolchuk VI, Wairkar YP, Jiao W, Evergren E, Pan H, Zhou Y, Venken KJ, Shupliakov O, Robinson IM, O’Kane CJ, Bellen HJ (2007) Eps15 and Dap160 control synaptic vesicle membrane retrieval and synapse development. J Cell Biol 178:309-322. CrossRef Medline

Korolchuk VI, Schütz MM, Gómez-Llorente C, Rocha J, Lansu NR, Collins SM, Wairkar YP, Robinson IM, O’Kane CJ (2007) Drosophila Vps35 function is necessary for normal endocytic trafficking and actin cytoskeleton organisation. J Cell Sci 120:4367-4376. CrossRef Medline

Lee CY, Wilkinson BD, Siegrist SE, Wharton RP, Doe CQ (2006) Brat is a Miranda cargo protein that promotes neuronal differentiation and inhibits neuroblast self-renewal. Dev Cell 10:441-449. CrossRef Medline

Lee J, Wu CF (2010) Orchestration of stepwise synaptic growth by K+ and $\mathrm{Ca}^{2+}$ channels in Drosophila. J Neurosci 30:15821-15833. CrossRef Medline

Li J, Ashley J, Budnik V, Bhat MA (2007) Crucial role of Drosophila neurexin in proper active zone apposition to postsynaptic densities, synaptic growth, and synaptic transmission. Neuron 55:741-755. CrossRef Medline
Liu Z, Huang Y, Zhang Y, Chen D, Zhang YQ (2011) Drosophila acyl-CoA synthetase long-chain family member 4 regulates axonal transport of synaptic vesicles and is required for synaptic development and transmission. J Neurosci 31:2052-2063. CrossRef Medline

Liu Z, Chen Y, Wang D, Wang S, Zhang YQ (2010) Distinct presynaptic and postsynaptic dismantling processes of Drosophila neuromuscular junctions during metamorphosis. J Neurosci 30:11624-11634. CrossRef Medline

Marie B, Sweeney ST, Poskanzer KE, Roos J, Kelly RB, Davis GW (2004) Dap160/intersectin scaffolds the periactive zone to achieve high-fidelity endocytosis and normal synaptic growth. Neuron 43:207-219. CrossRef Medline

Martin AR (1955) A further study of the statistic composition of the endplate potential. J Physiol 130:114-122. Medline

Menon KP, Sanyal S, Habara Y, Sanchez R, Wharton RP, Ramaswami M, Zinn K (2004) The translational repressor pumilio regulates presynaptic morphology and controls postsynaptic accumulation of translation factor elF-4E. Neuron 44:663-676. CrossRef Medline

Menon KP, Andrews S, Murthy M, Gavis ER, Zinn K (2009) The translational repressors Nanos and Pumilio have divergent effects on presynaptic terminal growth and postsynaptic glutamate receptor subunit composition. J Neurosci 29:5558-5572. CrossRef Medline

Mosca TJ, Hong W, Dani VS, Favaloro V, Luo L (2012) Trans-synaptic Teneurin signalling in neuromuscular synapse organization and target choice. Nature 484:237-241. CrossRef Medline

Muraro NI, Weston AJ, Gerber AP, Luschnig S, Moffat KG, Baines RA (2008) Pumilio binds para mRNA and requires Nanos and brat to regulate sodium current in Drosophila motoneurons. J Neurosci 28 : 2099-2109. CrossRef Medline

Nahm M, Kim S, Paik SK, Lee M, Lee S, Lee ZH, Kim J, Lee D, Bae YC (2010a) dCIP4 (Drosophila Cdc42-interacting protein 4) restrains synaptic growth by inhibiting the secretion of the retrograde glass bottom boat signal. J Neurosci 30:8138-8150. CrossRef Medline

Nahm M, Long AA, Paik SK, Kim S, Bae YC, Broadie K, Lee S (2010b) The Cdc42-selective GAP rich regulates postsynaptic development and retrograde BMP transsynaptic signaling. J Cell Biol 191:661-675. CrossRef Medline

O'Connor-Giles KM, Ho LL, Ganetzky B (2008) Nervous wreck interacts with thickveins and the endocytic machinery to attenuate retrograde BMP signaling during synaptic growth. Neuron 58:507-518. CrossRef Medline

Olesnicky EC, Bhogal B, Gavis ER (2012) Combinatorial use of translational cofactors for cell type-specific regulation during neuronal morphogenesis in Drosophila. Dev Biol 365:208-218. CrossRef Medline

Persson U, Izumi H, Souchelnytskyi S, Itoh S, Grimsby S, Engström U, Heldin CH, Funa K, ten Dijke P (1998) The L45 loop in type I receptors for TGF- $\beta$ family members is a critical determinant in specifying Smad isoform activation. FEBS Lett 434:83-87. CrossRef Medline

Pielage J, Fetter RD, Davis GW (2006) A postsynaptic spectrin scaffold defines active zone size, spacing, and efficacy at the Drosophila neuromuscular junction. J Cell Biol 175:491-503. CrossRef Medline

Qin G, Schwarz T, Kittel RJ, Schmid A, Rasse TM, Kappei D, Ponimaskin E, Heckmann M, Sigrist SJ (2005) Four different subunits are essential for expressing the synaptic glutamate receptor at neuromuscular junctions of Drosophila. J Neurosci 25:3209-3218. CrossRef Medline

Rodal AA, Motola-Barnes RN, Littleton JT (2008) Nervous wreck and Cdc42 cooperate to regulate endocytic actin assembly during synaptic growth. J Neurosci 28:8316-8325. CrossRef Medline

Schulte J, Sepp KJ, Jorquera RA, Wu C, Song Y, Hong P, Littleton JT (2010) DMob4/Phocein regulates synapse formation, axonal transport, and microtubule organization. J Neurosci 30:5189-5203. CrossRef Medline

Sonoda J, Wharton RP (2001) Drosophila brain tumor is a translational repressor. Genes Dev 15:762-773. CrossRef Medline

Stefanatos RK, Vidal M (2011) Tumor invasion and metastasis in Drosophila: a bold past, a bright future. J Genet Genomics 38:431-438. CrossRef Medline

Sweeney ST, Davis GW (2002) Unrestricted synaptic growth in spinster-a late endosomal protein implicated in TGF-beta-mediated synaptic growth regulation. Neuron 36:403-416. CrossRef Medline

Takaesu NT, Herbig E, Zhitomersky D, O’Connor MB, Newfeld SJ (2005) DNA-binding domain mutations in SMAD genes yield dominantnegative proteins or a neomorphic protein that can activate WG target genes in Drosophila. Development 132:4883-4894. CrossRef Medline 
Verstreken P, Kjaerulff O, Lloyd TE, Atkinson R, Zhou Y, Meinertzhagen IA, Bellen HJ (2002) Endophilin mutations block clathrin-mediated endocytosis but not neurotransmitter release. Cell 109:101-112. CrossRef Medline

Verstreken P, Ohyama T, Bellen HJ (2008) FM 1-43 labeling of synaptic vesicle pools at the Drosophila neuromuscular junction. Methods Mol Biol 440:349-369. CrossRef Medline

Verstreken P, Ohyama T, Haueter C, Habets RL, Lin YQ, Swan LE, Ly CV, Venken KJ, De Camilli P, Bellen HJ (2009) Tweek, an evolutionarily conserved protein, is required for synaptic vesicle recycling. Neuron 63: 203-215. CrossRef Medline

Wang D, Zhang L, Zhao G, Wahlstrom G, Heino TI, Chen J, Zhang YQ (2010) Drosophila twinfilin is required for cell migration and synaptic endocytosis. J Cell Sci 123:1546-1556. CrossRef Medline

Wang X, Shaw WR, Tsang HT, Reid E, O'Kane CJ (2007) Drosophila spichthyin inhibits BMP signaling and regulates synaptic growth and axonal microtubules. Nat Neurosci 10:177-185. CrossRef Medline
Wucherpfennig T, Wilsch-Bräuninger M, González-Gaitan M (2003) Role of Drosophila Rab5 during endosomal trafficking at the synapse and evoked neurotransmitter release. J Cell Biol 161:609-624. CrossRef Medline

Yao A, Jin S, Li X, Liu Z, Ma X, Tang J, Zhang YQ (2011) Drosophila FMRP regulates microtubule network formation and axonal transport of mitochondria. Hum Mol Genet 20:51-63. CrossRef Medline

Yao CK, Lin YQ, Ly CV, Ohyama T, Haueter CM, Moiseenkova-Bell VY, Wensel TG, Bellen HJ (2009) A synaptic vesicle-associated $\mathrm{Ca}^{2+}$ channel promotes endocytosis and couples exocytosis to endocytosis. Cell 138:947-960. CrossRef Medline

Zhang B, Koh YH, Beckstead RB, Budnik V, Ganetzky B, Bellen HJ (1998) Synaptic vesicle size and number are regulated by a clathrin adaptor protein required for endocytosis. Neuron 21:1465-1475. CrossRef Medline

Zhao L, Wang D, Wang Q, Rodal AA, Zhang YQ (2013) Drosophila cyfip regulates synaptic development and endocytosis by suppressing filamentous actin assembly. PLoS Genet 9:e1003450. CrossRef Medline 